\title{
Research Paper \\ Time Usage Patterns of Iranian Older Adults and Its Association With Socioeconomic Factors
}

\author{
Maryam Sharifian Sani ${ }^{1,2},{ }^{*}$ Nasibeh Zanjari ${ }^{2}$, Rasoul Sadeghi ${ }^{3}$
}

1. Department of Social Welfare, University of Social Welfare and Rehabilitation Sciences, Tehran, Iran.

2. Crawford School of Public Policy, College of Asia and the Pacific, Australian National University, Australia.

3. Department of Demography, Faculty of Social Sciences, University of Tehran, Tehran, Iran.

Citation: Sharifian Sani M, Zanjari N, Sadeghi R. [Time Usage Patterns of Iranian Older Adults and Its Association With Socioeconomic Factors (Persian)]. Iranian Journal of Ageing. 2016; 11(3):400-415. http://dx.doi.org/10.21859/sija-1103400

dof : $:$ http://dx.doi.org/10.21859/sija-1103400

Received: 20 Apr. 2016

Accepted: 22 Agu. 2016

Key words:

Time use, Leisure,

Older adults, Qual-

ity of life, Iran

\section{ABSTRACT}

Objectives Time usage patterns of older adults is an important indicator of their health and quality of life. This study aimed to assess the time usage patterns of Iranian older adults and its association with social-demographic factor.

Methods \& Materials This study was based on the secondary analysis of time use data of Iranians. The data were collected by questionnaire based on stratified sampling by Statistical Center of Iran in 2009. The sample size comprised 3516 Iranian elderly (60 years and older) who live in urban areas. The Chisquare, ANOVA, and multiple classification analysis (MCA) were used for data analysis.

Results The results indicated that Iranian older adults allocated the highest average time on activities related to personal care, leisure, unpaid works, religious activities, voluntary social activities, and paid works. The results also showed differential time usage patterns among different socioeconomic groups. Conclusion Multivariate analysis indicated that age, gender, activity status, education level, living arrangement, and disability status are most important variables in the prediction of time use patterns.

\section{* Corresponding Author:}

Address: Iranian Research Center on Ageing, University of Social Welfare and Rehabilitation Sciences, Koodakyar Ave., Daneshjoo Blvd., Evin,Tehran, Iran. Tel: +98 (21) 22180048

E-mail: zanajari.nz@gmail.com 


\title{
الكوهاى تَذران وقت سالمندان ايرانى و همبستههاى اقتصادى اجتماعى آن
}

\author{
مريم شريفيانثانى" "، "نسيبه زنجرى"؟ رسول صادقى"

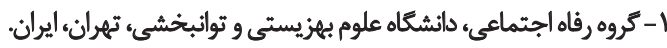

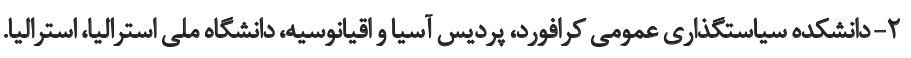

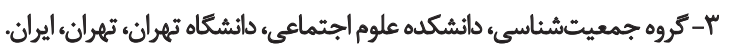

\begin{abstract}
حكند

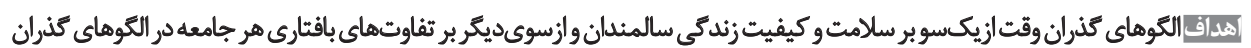

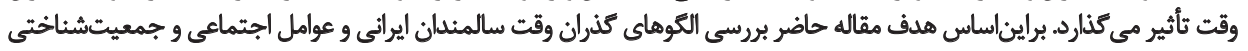

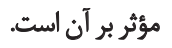

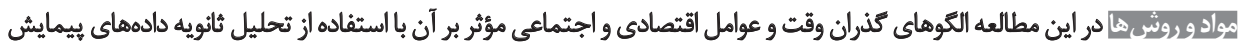

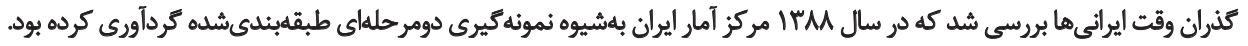

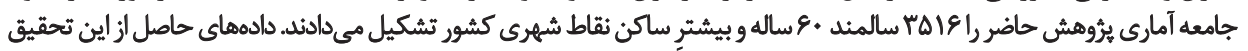

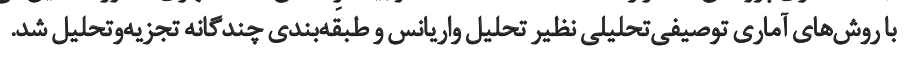

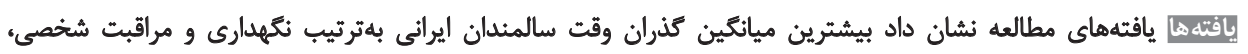

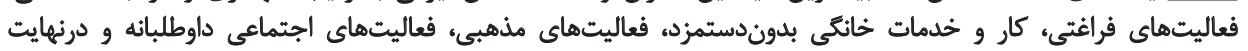

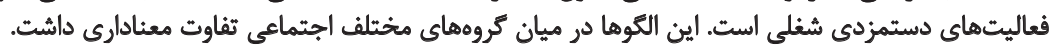

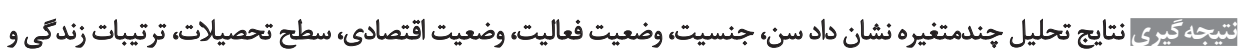

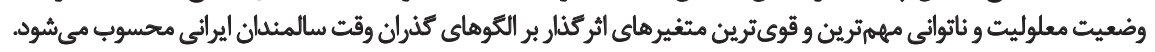

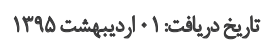

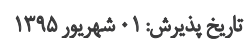

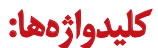
كذران وقت، اوقات فراغت،

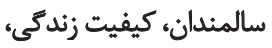
ايران
زمان يكى از مهامترين منابع براى هر فرد و جزء منابع محدود

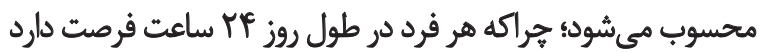

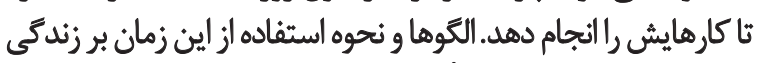

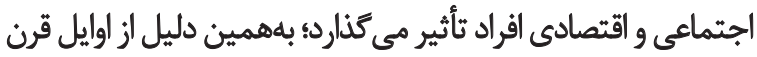

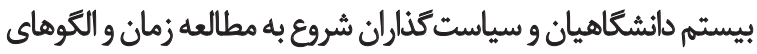

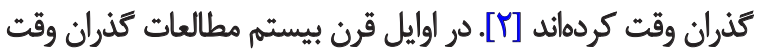

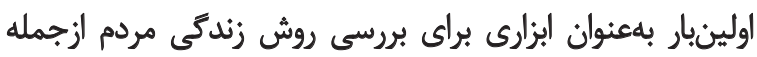

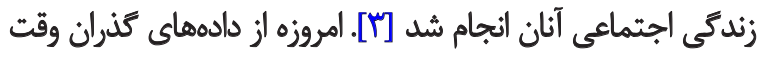

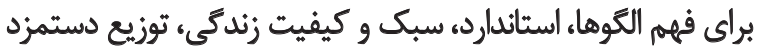

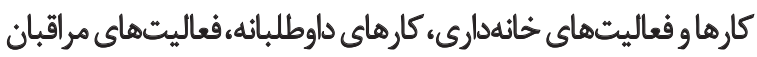

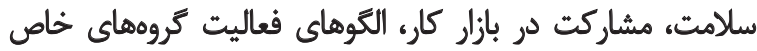

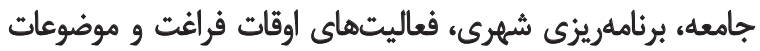

توسعه ملى استفاده مى شيود [f]

التوهاى تذران وقت در هر فرهنگ و بافتارى مثفاوت است؛

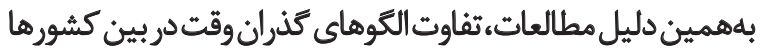
و كروهاى مختلف اجتماعى يك كشور را مقايسه كردهاند [ه، ؟). doles

باتوجهبه طولانى شدن عمر و سيرىشدن بخش درخورتوجهيى

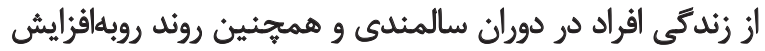

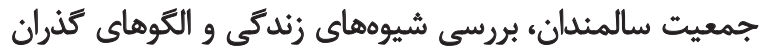

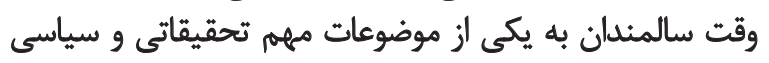

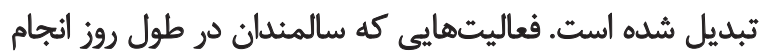

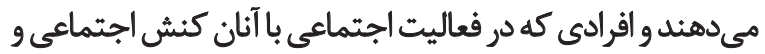

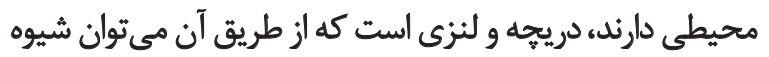

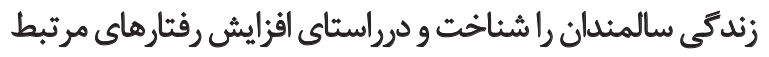
باسلامت برنامعريزى كرد [1]].

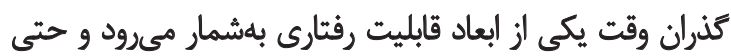

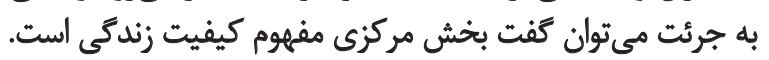

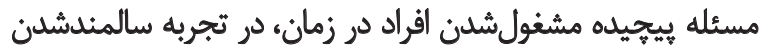

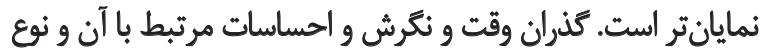

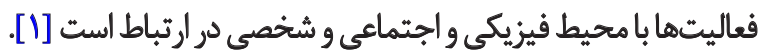

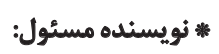
دكتر نسيبه زنجرى نوينديه

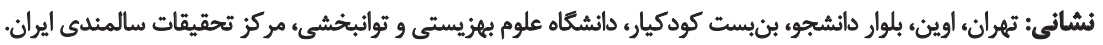

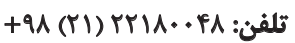
يست الكترونيكي: zanajari.nz@gmail.com 
شده است [•1]. در جامعهشناسى كلاسيك در توضيح الكوى

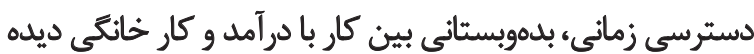

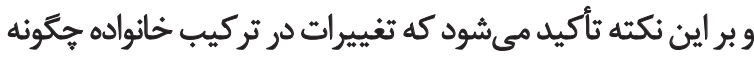

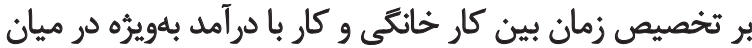

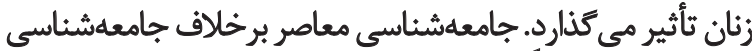

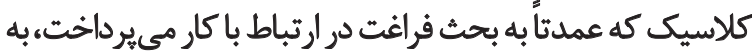

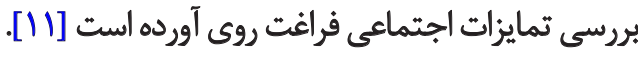
درزمينه الكوهاي كذران وقت مطالعات متعددى در كشورهاى

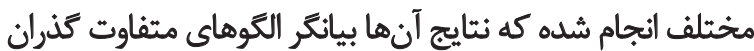

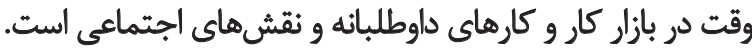

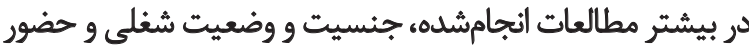

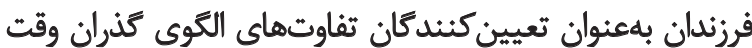

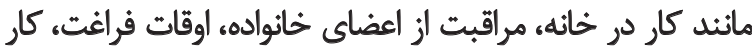

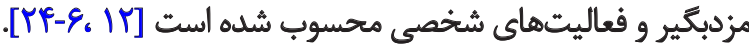

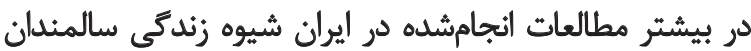

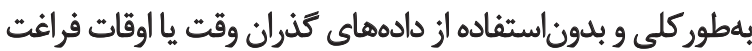

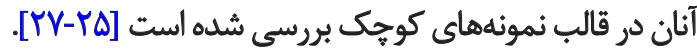

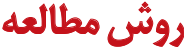

روش اين يُوهش توصيفىتحليلى بود كه در آن دادهايى

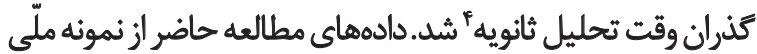

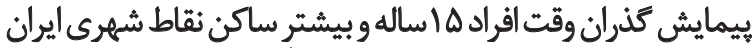

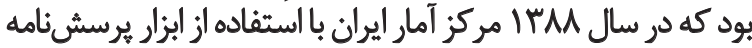

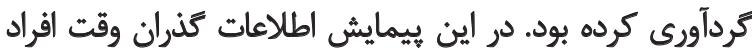

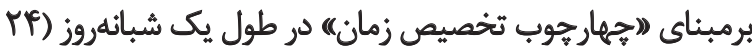

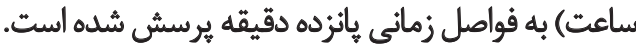
تعداد نمونههاى اين تحقيق

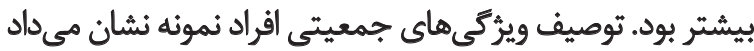

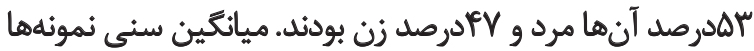
99/D سال محاسبه شد. حدود انهاد

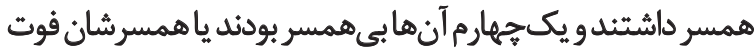

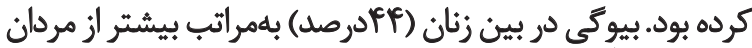

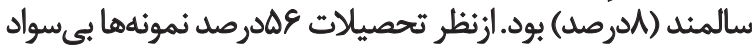

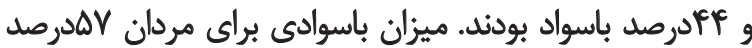

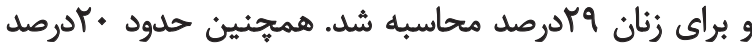

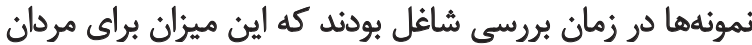

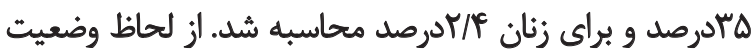

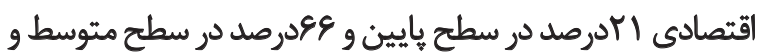
rا إدرصد در سطح مرفه قرار داشتيند.

در اين مطالعه الكَوهاي كذران وقت سالمندان در شش مقوله

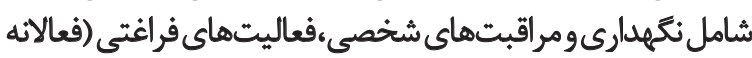

4. Secondary analysis
الكوى كذران وقت براساس هرخه زندكى تغيير مى كند؛ ازاينرو

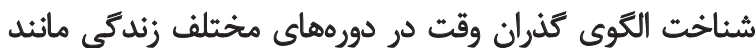

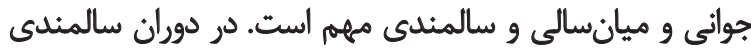

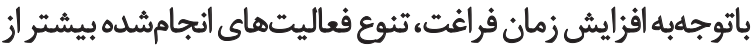

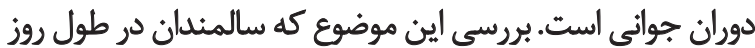

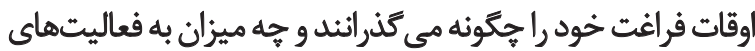
فيزيكى و اجتماعى مى يردازند، براى محققان و سياست

اين عرصه كمك كنثنه خواهد بود [ن]

بدينترتيب باتوجهبه اهميت مطالعه و بررسى التوهاي كذران

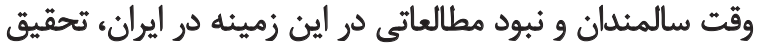

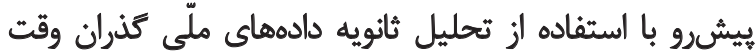

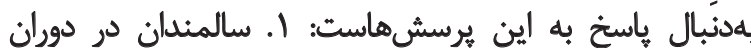

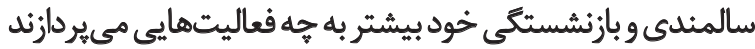

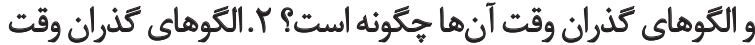

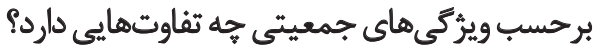

درزمينه تخصيص زمان، نظريهها و الكوهاى مختلفى مطرح شده

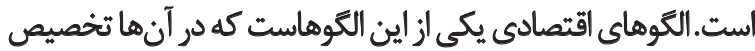

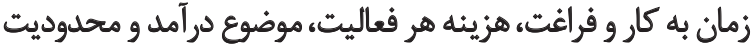

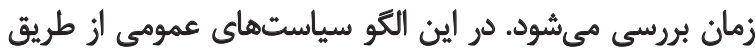

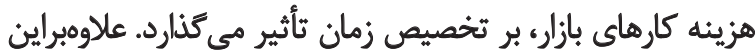

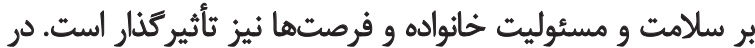

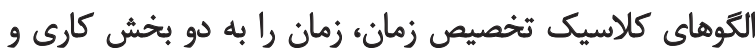

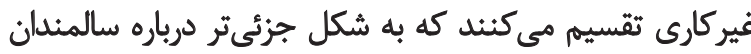

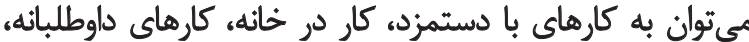

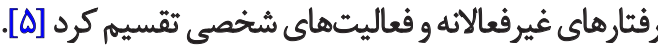
نظريه تخصيص زمان' در اقتصاد سه استفاده اصلى از زمان را را

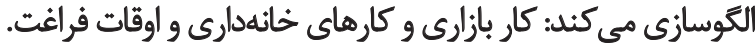

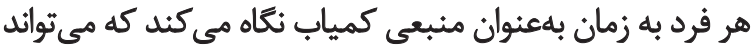

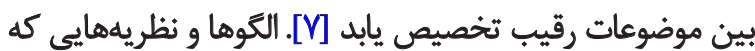

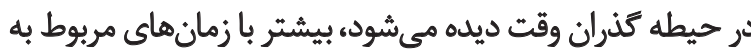

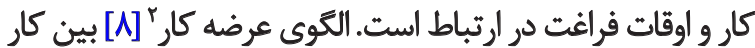

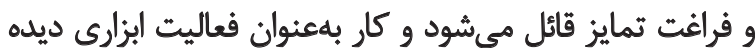

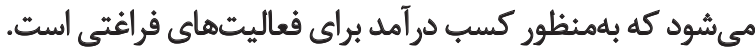

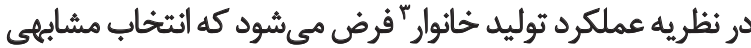

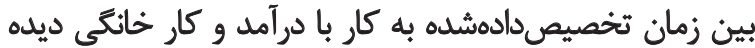

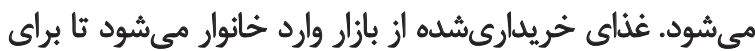

مصرف نهايى آماده شود [9].

علاومبر ديدگًاه اقتصادى در جامعهشناسى نيز الكوهاى گذذران

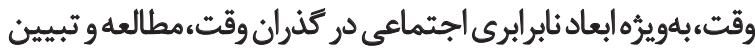

1. Time allocation

2. Labor supply

3. Household production function 
براى تجزيهوتحليل دادهها از روشهاى آمار توصيفىتحليلى

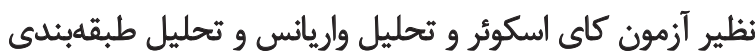
جندكانه (MCA) إستفاده شد.

يافتهها

الكوهاى كذران وقت سالمندان در شش مقوله بررسى و در

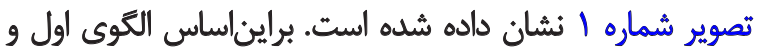

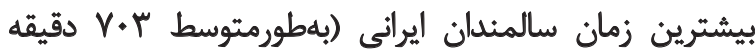

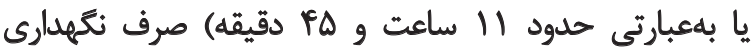

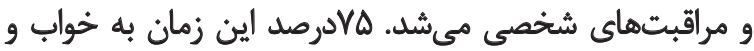

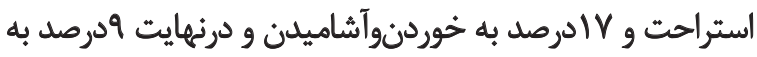
مراقبت و بهداشت فردى تعلق مي خيرد.

الكوى دوم تذران وقت، فعاليتهاى فراغتى را شامل مى بشد

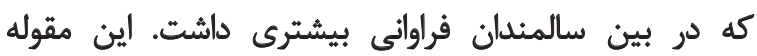

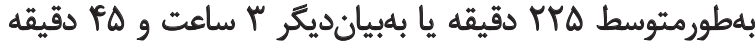

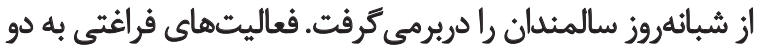

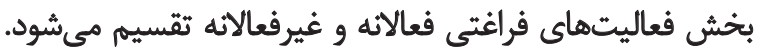

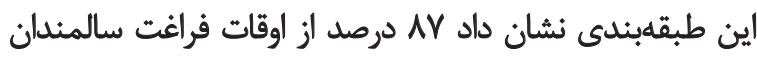

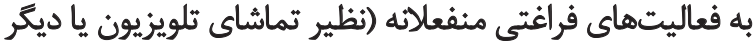

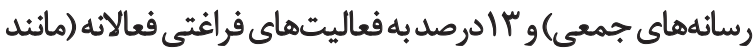

5. Multiple Classification Analysis (MCA)

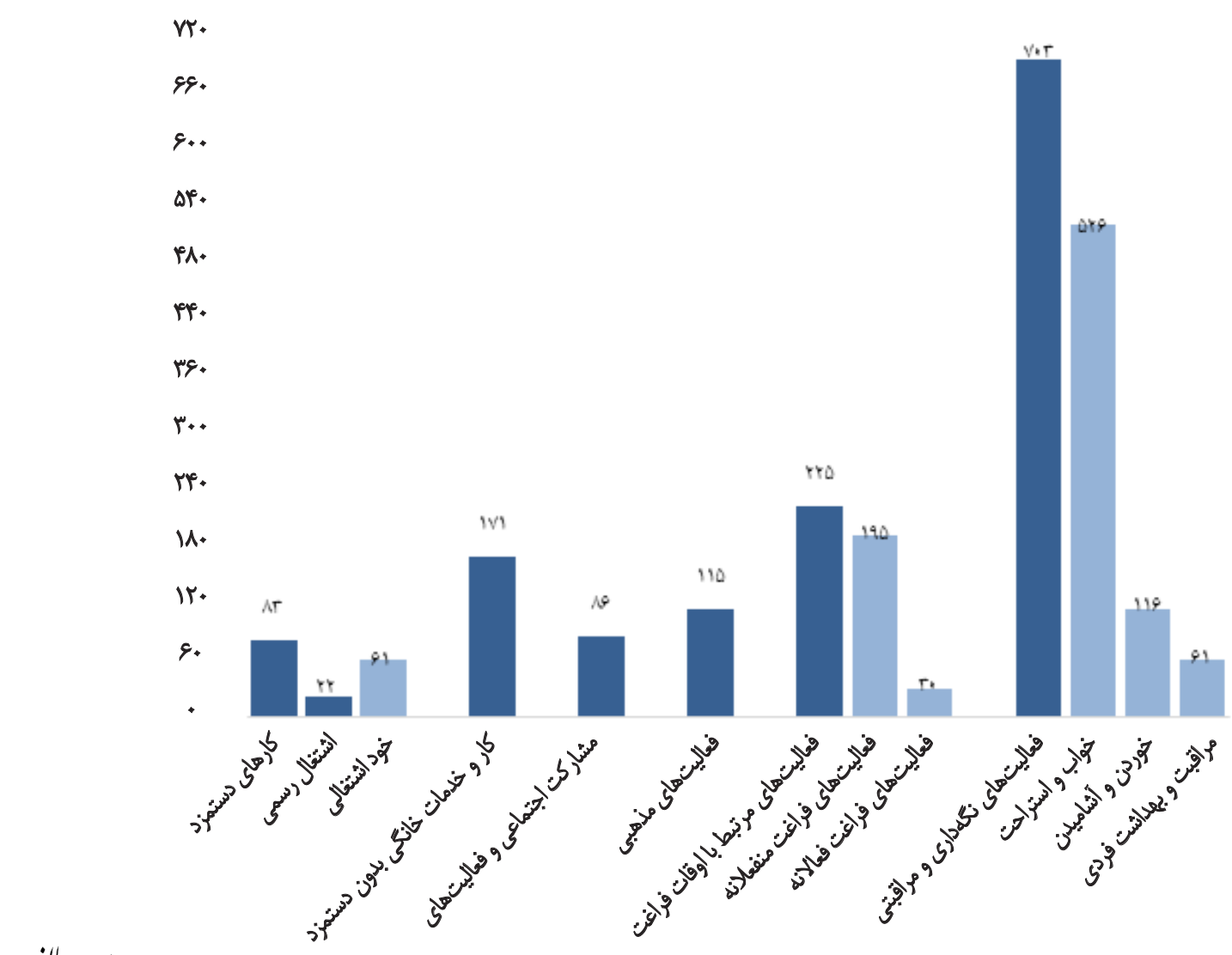

و غيرفعالانه)، كار و خدمات خانكى بدوندستمزد، فعاليتهاي

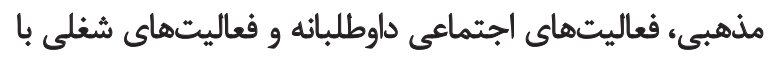

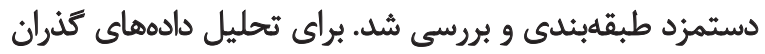
وقت سه مفهوم كليدى بايد مدنظر قرار كيرد: بردي 1.فعاليت اوليه: فعاليت اصلى كه در زمان مشخصى انجام مىشود؛

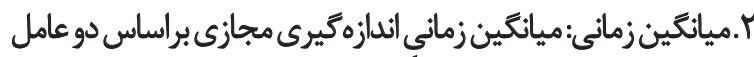

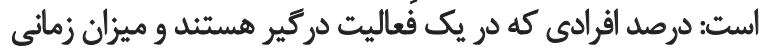

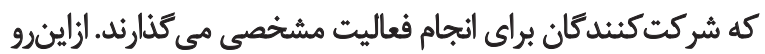

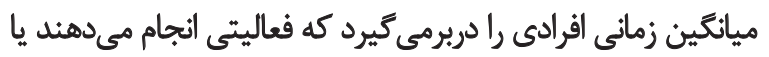

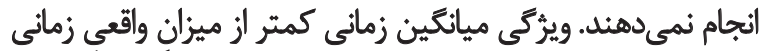

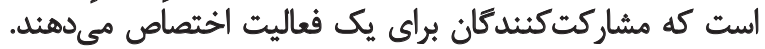

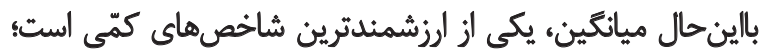

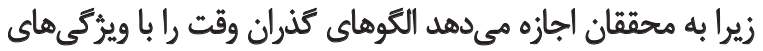

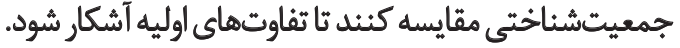

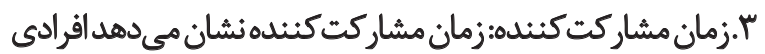

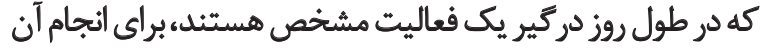

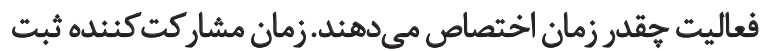

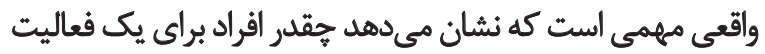

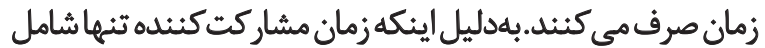

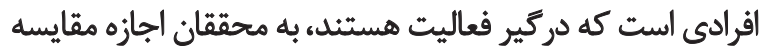

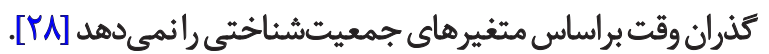

تصوير ا. التوهاى كندران وقت سالمئدان •هُاله و بيشتر در مناطق شيهرى ايران. 
جدول ا. نسبت حضور و مشاركت سالمثدان در هريك از الكوهاي كندران وقت بهتفكيك جنسيت.

\begin{tabular}{|c|c|c|c|c|c|c|c|c|c|}
\hline \multicolumn{6}{|c|}{ متوسط زمان صرفشده (به دقيقه) براى افراد دريّير در آن فعاليت } & \multirow{3}{*}{ زن } & \multirow{3}{*}{ مرد } & \multirow{3}{*}{ هن هر } & \multirow{3}{*}{ 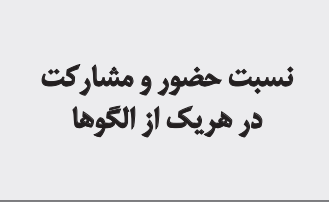 } \\
\hline \multicolumn{2}{|c|}{ زن } & \multicolumn{2}{|c|}{ 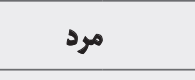 } & \multicolumn{2}{|c|}{ هر دو جنس } & & & & \\
\hline تعد/اد & زمان & تعداد & زمان & تعداد & زمان & & & & \\
\hline ine & Ma & gres & f.1 & Ar. & $r \Delta$ & 1.10 & $M \varphi / N$ & $m$ & كارهاي دستمزي \\
\hline PA & TVD & rie & (q) & repe & rM & $r / 9$ & $11 / 8$ & $V / \Delta$ & أشتغال ربسمى \\
\hline iv & irq & DIV & r. & exp & rro & Na & $r V / A$ & Wr & خوداشتعالى \\
\hline $10 \cdot \%$ & ra. & $|r q|$ & if. & TMas & MIO & $9 . / 9$ & $\varepsilon q / F$ & $r q / \Delta$ & كار وخلدمات خانكي بلوندستمزد \\
\hline $1 r+1$ & irf & ITH & IMP & MPAI & inf & $n \%$ & $e q / 1$ & eq/ & هشاركت اجتماعى و فعاليتهاي \\
\hline IOTM & irq & IFNA & IrT & $M+\Lambda$ & Ire & $9 r /$. & $9 \cdot 10$ & $91 / \%$ & فعاليتهاى مذهبى \\
\hline$|\Delta|^{\prime}$ & MI9 & $i m+$ & raf & $M+\Delta$ & rmq & $\$ Y / \Delta$ & $9 \Delta / 4$ & qf\%. & فعاليتهاي مرثبط با اوقات فراغت \\
\hline $10 \cdot 4$ & $r+g$ & $\ln$ & MIE & me & rit & 9.11 & $9 m / 1$ & $9 \mathrm{~s} /$. & فعاليتهاى فراغتى منفعلائه \\
\hline mI & $\Delta \mu$ & VDE & 1.9 & $1.9 V$ & $1 .$. & WA & $p+18$ & $r+/ r$ & فعاليتهاى فراغتى فعالانه \\
\hline \multirow[t]{2}{*}{ IEAD } & $v+1$ & $|w|$ & $V \cdot \Delta$ & rals & $V \cdot r$ & & & & فعاليتهاى نتههدارى و مراقبتى \\
\hline & & & & & & $1 . . \%$ & $1 . . \%$ & $1 . .1$ & شخصى \\
\hline IEAf & ArT & We. & ar. & raiff & are & $99 / 9$ & $99 / 9$ & 9ข/9 & خواب و استراجت \\
\hline IFta & 110 & Was & IIV & $r a \cdot f^{f}$ & 118 & $99 / 7$ & $99 / 8$ & $94 /$ & خورنو آشاميلن \\
\hline 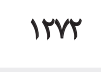 & v. & $18 \cdot 1$ & ra & rAVT & VD & $v e / q$ & $\Lambda ه / V$ & $A I N$ & هراقبت و بهد|نشت فردى \\
\hline $1 \Leftarrow \Delta \Delta$ & - & $|W|$ & - & role & - & $1 F \Delta \Delta$ & $|w|$ & role & تعداد نمونه \\
\hline
\end{tabular}

攵

فعاليتهاي داوطلبانهمير براختند.

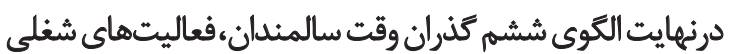

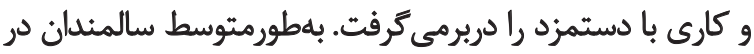

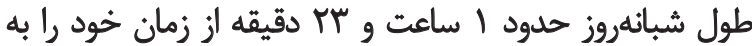

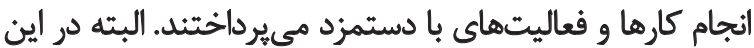

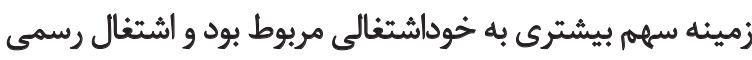

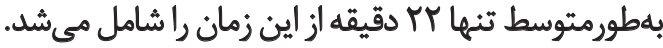
بررسى نسبت حضور و مشاركت سالمندان در هريك از الكوهاى

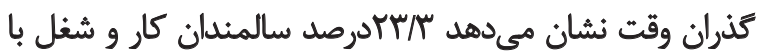

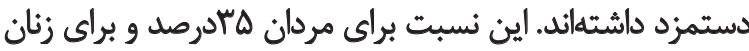

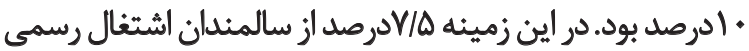

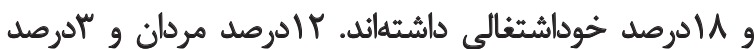

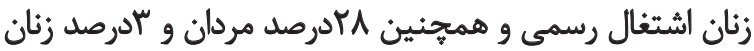

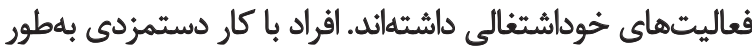

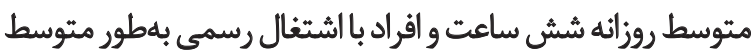

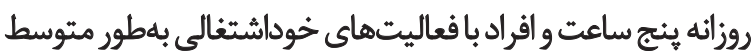

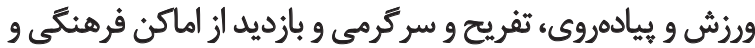

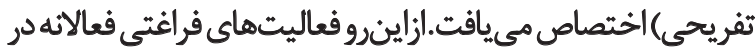

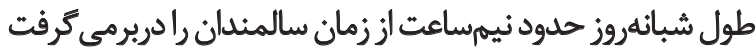

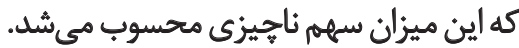
الكوى سوم كذران وقت سالمندان، كار و خدمات خانكى

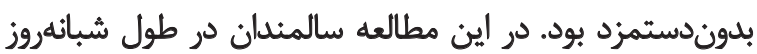

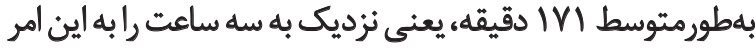

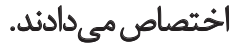

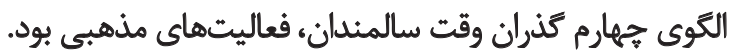

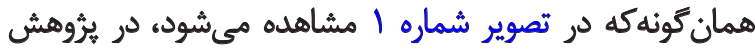

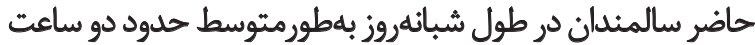
به عبادت وفعاليتهاى مذهبى مي يرداختيند.

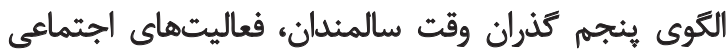

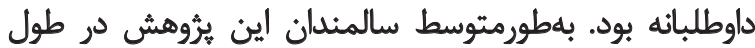

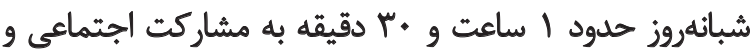


جدول r. همبستههاى اقتصادىاجتماعى زمان اختصاصيافته به كارهاى دستمزدى و خانتى (نتايج آزمون MCA).

\begin{tabular}{|c|c|c|c|c|c|c|c|c|c|c|}
\hline \multirow[b]{2}{*}{ تمونه } & \multicolumn{2}{|c|}{ كار خانكي بدوندستمزد } & \multicolumn{2}{|c|}{ خوداششتغالى } & \multicolumn{2}{|c|}{ أشتغال رسمى } & \multicolumn{2}{|c|}{ كار دستمزدى } & & \\
\hline & 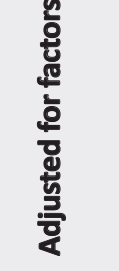 & 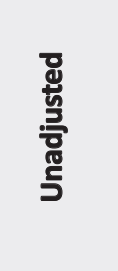 & 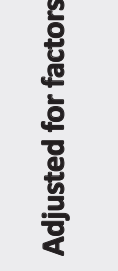 & 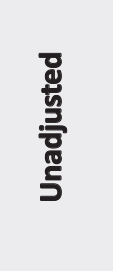 & 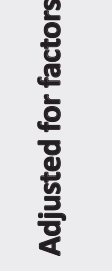 & 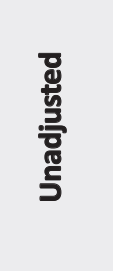 & 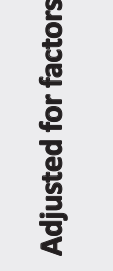 & 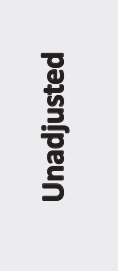 & & \\
\hline$|N|$ & $119 / 1$ & $q \varepsilon / q$ & $\varepsilon \Delta / r$ & $1 \cdot \Delta / 4$ & $19 / 4$ & $M / A$ & $\lambda F / \Delta$ & $1149 / \pi$ & هرد & \\
\hline $1 \notin \Delta \Delta$ & TrN/N & $r \Delta f /$. & $\Delta \& / q$ & $11 / 0$ & $M H / T$ & $N$ & A)/ & $19 / 0$ & زن - ت & جنسيت \\
\hline- & $+M+* * *$ &.$/ 49$ &.$/ .48$ & $+/ \pi 90^{\circ * *}$ & . &.$/ 1 \Lambda^{* 0 *}$ & $+1+.9$ & $\cdot / \pi T \Lambda^{* * *}$ & اثاو بتا & \\
\hline $111 \%$ & IAV/S & $14 \mathrm{Ne}^{e}$ & $E V / \Delta$ & $A T / T$ & $M T / N$ & rV/a & $9 . / r$ & $11 \%$ & 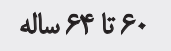 & \\
\hline vAr & $\mid A \Psi / A$ & $\mid A \Delta /$. & $81 / 5$ & $V / / \Delta$ & $M / \Delta$ & TNY & $N \otimes / N$ & $1 \cdot 1 / \mathrm{N}$ & 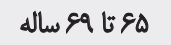 & \\
\hline hev & $1 E V / T$ & $181 / 9$ & $\Delta N Y$ & $\Delta r / P$ & $W^{W}$ & $1 \& / f$ & $V \& / \Delta$ & $89 / 1$ & 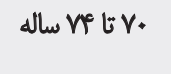 & كروهالي \\
\hline$P q D$ & $10 \cdot / 9$ & IPA/S & $\Delta \Delta / q$ & rNE & $I V / P$ & $1 \% /$. & $n / r$ & $\Delta \cdot / N$ & 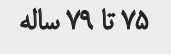 & سنى \\
\hline rvq & ITNK & $111 / 8$ & $\Delta \& / K^{m}$ & $19 / 9$ & $M / A$ & $1 Y / 8$ & $A M / 1$ & $M T / \Delta$ & 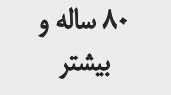 & \\
\hline - & •/NTrmete &.$/ 1 V \Delta^{* * *}$ &.$/ .49$ & */NTrene* & $. / . \mu+$ & $+1+v g * *$ &.$/ .4 \Delta^{\circ}$ & t/Nareses & اتثاو بتا & \\
\hline TAM & IV9/9 & IEg/ & $91 / 9$ & VN. & $M T / A$ & $r \varepsilon / \Gamma$ & 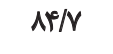 & $1.8 / T$ & داراى همسر & \\
\hline MF & $\mid f \omega / 1$ & MF/q & 8.11 & $I r / N$ & WE & $N T$ & VAN & $M / 9$ & بيوه & \\
\hline MT & $\mathrm{AV} / \mathrm{T}$ & $r \cdot 1 / 8$ & $9 x / 9$ & Q1/1 & $18 / 9$ & $19 / r$ & AI/A & $v \cdot / r$ & مطلقه & تأهل \\
\hline ir & SND & $90 / A$ & $I r / 8$ & $1 / r$ & $1 \mathrm{H} / \mathrm{S}$ & $10 /$ & $r \Delta / r$ & $18 / r$ & ازازواتهنكرده & \\
\hline- & $\cdot 1 \cdot+4=$ & $.1 .81^{* *}$ & $.1 \cdot 19$ & ./NYeren &. $\mid+r$ & -1*Apen &.$/ . M P$ &.$/ 19 V^{* * * *}$ & اتأو بتا & \\
\hline 19VD & IEV/K & $|A| / 9$ & gQ/P & $\Delta \varphi /$. & $\mathrm{r} / \mathrm{.}$ & WA & NE/S & $V / \Delta$ & بىسواد & \\
\hline $9 \vee A$ & $\mid \mathrm{gal}$. & $\mid Q Y / *$ & $\Delta N \mid$ & $1 \cdot 19$ & WD & $r \in / \pi$ & vele & $1 . \% / 9$ & ابتلايي & \\
\hline Wr & $I V A / A$ & IENA & $e x /$. & $8 \cdot \pi$ & $M T / \Delta$ & $r+/ 1$ & $N / \Delta$ & $\lambda \cdot / r$ & راهنمايى & سطح \\
\hline MTA & $194 / r$ & $I V * / V$ & $\Delta \& / V$ & $\Delta+/ \pi$ & $r q / r$ & $r \Delta / r$ & $N \%$ & $V \Delta / \Delta$ & متوسطه / دييلم & تحصيلات \\
\hline lar & $1119 / 2$ & $\| \leftarrow \Delta / A$ & $r q / \mathrm{V}$ & $p q / 8$ & re/V & $P 1 / \Delta$ & $89 / f$ & $91 / \pi$ & دانشكاهى & \\
\hline- & $+1+\bullet \Lambda^{*}$ & $.1 \cdot M^{* * *}$ & $\cdot 1+4 \lambda^{* *}$ & $. / . \vee y=*$ & $.1+\mu f *$ &.$/ . \Delta F^{\circ}$ & $\cdot /\left.\cdot r\right|^{*}$ &.$/ \cdot \mathrm{VA}^{\infty}$ & اتاو بتا & \\
\hline SNA & $9 \pi / 1$ & sql. & YNST & rA\&/A & AVpe & NEIS & $M r / A$ & mepr & شاغل & \\
\hline$i f+F$ & $18 V / r$ & $\| F r / r$ & $8 / 1$ & $\Delta / N$ & $V / A$ & $V / I$ & $1 r / 9$ & $I r / A$ & با بادرن آمد & \\
\hline IIIT & rre/ & rAF/F & NE & $V / \Lambda$ & r/g & $p / f$ & $11 / r$ & $11 / 0$ & خانهدار & فعاليث \\
\hline rad & $\mid \Delta T / \Delta$ & $11 \cdot / r$ & $m$ & $M / T$ & $V / T^{M}$ & $\Delta N$ & $11 / 8$ & $N 9$ & ديكر & \\
\hline- & " Mqume* & . /Arr*a* & $.189 \Delta^{\circ * * *}$ & $+/ N \cdot 8+*$ & $+M+q * \cdots$ & . & $. / N A A^{\infty}=$ & + Mar & اتا و بتا & \\
\hline
\end{tabular}




\begin{tabular}{|c|c|c|c|c|c|c|c|c|c|c|}
\hline \multirow[b]{2}{*}{ تمونه } & \multicolumn{2}{|c|}{ كار خانئي بدوندستمزد } & \multicolumn{2}{|c|}{ مُوداششتغالِي } & \multicolumn{2}{|c|}{ أشنغال رسمي } & \multicolumn{2}{|c|}{ كار دستمزدي } & & \\
\hline & 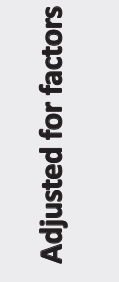 & 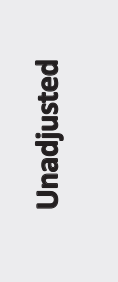 & 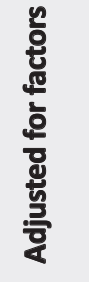 & 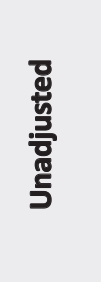 & 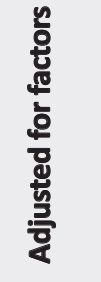 & 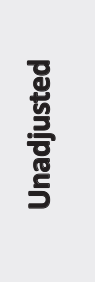 & 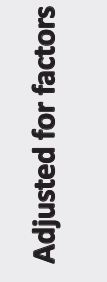 & 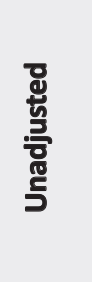 & & \\
\hline ner & $M T / q$ & $\mathrm{IV} / \mathrm{s}$ & $\Delta \Delta / \mathrm{V}$ & $\Delta f / q$ & $18 / \pi$ & $|V /|$ & $V .10$ & 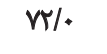 & بايين & \\
\hline MmT & $18 \mathrm{~V} / \mathrm{q}$ & $19 V / 9$ & $g / N$ & ED/F & $\pi /$ & $M r / q$ & $A \& \mathbb{A}$ & ND & متونيط & |قتصادي \\
\hline pie & IED/A & $M A / Y$ & $\Delta N Y$ & $P q / \Delta$ & $\mathrm{rel}$ & $M Y / A$ & Afr & $V / \pi$ & 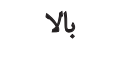 & خاتوار \\
\hline - & $.1 .4 . *$ & . & $+|+r|$ & .1 .49 & $.1+4 T^{*}$ & $.1 . r \Delta$ & 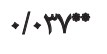 & $.1 \cdot 94$ & اتا و بثا & \\
\hline PVE & $I W / \pi$ & $\mathrm{IVI} / \mathrm{s}$ & $e r / 8$ & $\mu \varphi / T$ & $r+/ r$ & $M / / q$ & $9 r / 1$ & 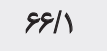 & تنها & \\
\hline$r+r$. & $1 E 9 / A$ & $I V \cdot N$ & $9 \cdot / 9$ & $89 \%$ & $r \cdot / r$ & $\mathrm{M} / \mathrm{s}$ & A)/ $/ \mathrm{r}$ & $\Lambda \otimes / \Delta$ & غيرتنها & تنهايیي \\
\hline- & .1 .18 & $\%$ & $.1 . .8$ & $\cdot 1 \cdot R T^{*}$ & $+1+\pi r^{*}$ & $+1+.1$ & 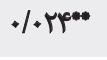 & 1.re* & ائا و بتا & \\
\hline ria & $1+9 / r$ & $9 \% / \%$ & $\Delta V / N^{\mu}$ & t/8 & $\pi r / r$ & $N V$ & $\mathrm{ra} / \mathrm{V}$ & $\mathrm{ir} / \mathrm{r}$ & بلى، دارد & نياز به \\
\hline$M+1$ & $\ln x / 9$ & IVD/9 & $81 / 8$ & $9 \Delta /$. & $\mathrm{rV} / \mathrm{g}$ & $M Y / \Delta$ & $A H / 1$ & $A V / \Delta$ & خير، نذارد & بهراقبتيت \\
\hline - & $.1 .99^{c * * *}$ & - Mrten** & $+1+.8$ & $.1 .9)^{* *}$ & $. / \cdot+r$ & $.1 \cdot r 0^{\circ}$ & 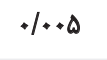 & $.1 .9 \gamma^{+\infty}$ & اتا و بتا & ن اتوائى \\
\hline Tale & \multicolumn{2}{|c|}{$\Lambda \cdot / r \Delta^{* \cdots *}$} & \multicolumn{2}{|c|}{$|\Delta r / \Lambda|^{20 * * *}$} & \multicolumn{2}{|c|}{ TI/EV**: } & \multicolumn{2}{|c|}{ roq/quen } & \multicolumn{2}{|c|}{ F Model } \\
\hline
\end{tabular}

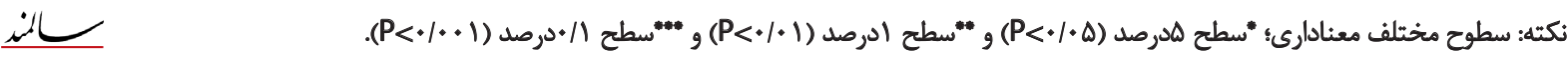

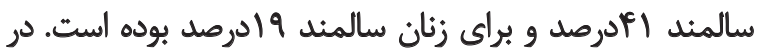

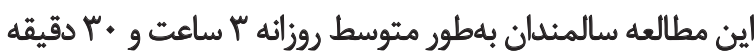

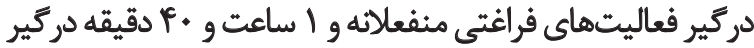
فعاليتهاى فراغتى فعالانه بودهاند.

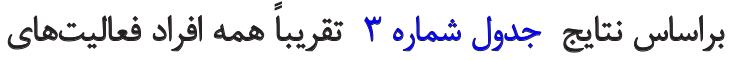

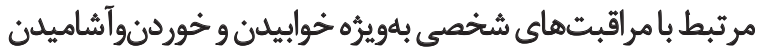

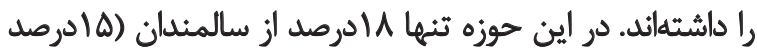

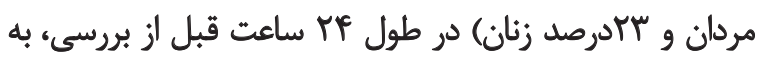
بهداشت و مراقبت شخصى نيرداختهاند.

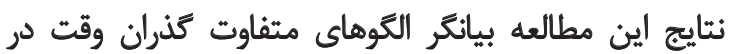

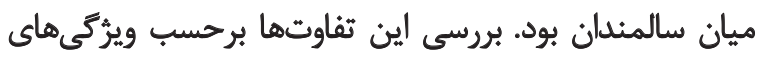

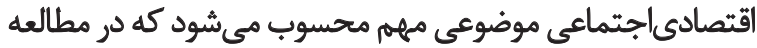
حاضر با استفاده از آزمون خُخدمتغيرى MCA بروسى شده است.

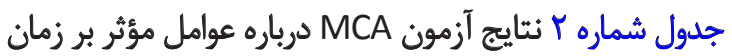

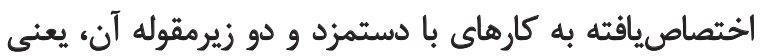

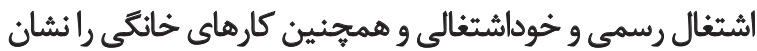

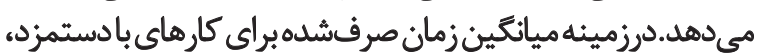

روزانه جهار ثا ينج ساعت كار مىكردند. زمان اختصاصيافته

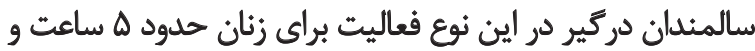
مردان r ساعت و •r دقيقه بود. همانطوركه در جدول شماره ا مشخص است، حدود • مدرصد

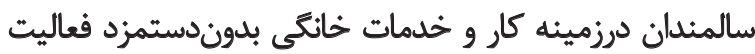

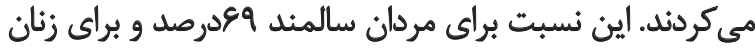

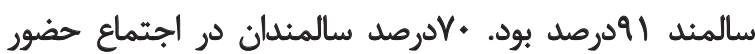

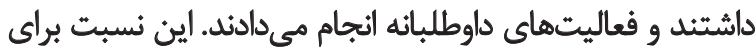

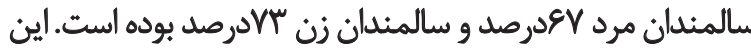

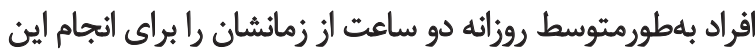

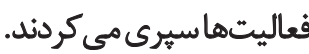

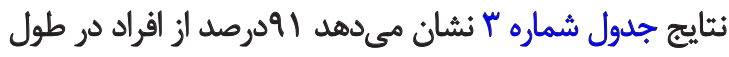

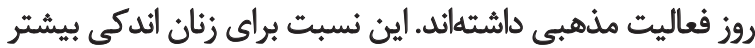

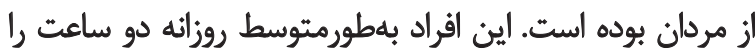

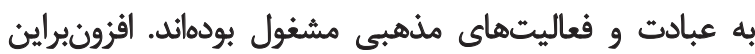

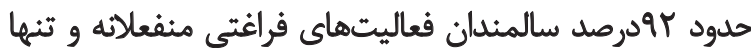

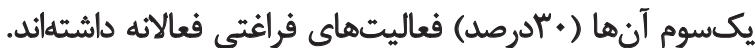

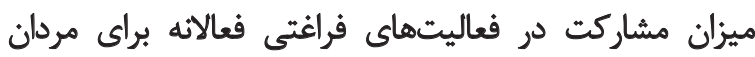


جدول r. هميستهاي اقتصادىاجتماعى زمان اختصاصيافته به مشاركت اجتماعى و فعاليتهاى داوطلبائه و مذهبى (نتايج آزمون MCA).

\begin{tabular}{|c|c|c|c|c|c|c|}
\hline \multirow{2}{*}{ تعداد نمونه } & \multicolumn{2}{|c|}{ فعاليتهاى مذهبى } & \multicolumn{2}{|c|}{ مشاركت اجتماعى و فعاليتهاى } & & \\
\hline & $\begin{array}{l}\text { Adjusted for } \\
\text { Factors }\end{array}$ & Unadjusted & $\begin{array}{l}\text { Adjusted for } \\
\text { Factors }\end{array}$ & Unadjusted & & \\
\hline$|A|$ & $11 \mathrm{~V} /$ & $11 \cdot 18$ & NE/8 & $A T / Q$ & مرد & \\
\hline $18 \Delta \Delta$ & $I 1 Y /$ & $119 / \pi$ & $N \& / T$ & $9 . / 2$ & نs & جنسيت \\
\hline- & $.1+\mathrm{rr}$ & $.1 . p V^{* *}$ & $.1 .0 r$ & $.1 .49 *$ & اتثا و بتا & \\
\hline$M \pi r$ & IIY/E & $111 / 4$ & $\Lambda \Delta / r$ & A)/8 & 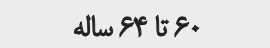 & \\
\hline var & $11 \Delta / \Delta$ & $11 \% / 9$ & $A r / 1$ & $A Y \%$ & هي & \\
\hline ner & $M \cdot / F$ & $1 T \cdot 10$ & $9 r / \Delta$ & $94 / 0$ & ساله Vi V. & \\
\hline 190 & $11 \% / \Delta$ & $\| 10 / 8$ & $\lambda F / q$ & NWI & ساله V9 Vه & كروهماي ستى \\
\hline ma & $1+9 / 1$ & $11+N$ & $N E / F$ & $9 \cdot \pi$ & •م ساله و بيشتر & \\
\hline- &.$/ . r A$ & $+1+49$ & מם & $\circ / \Delta \Delta \sigma^{*}$ & اثتا و بتا & \\
\hline TAM & $\| I r / r$ & $111 / 8$ & NA & $A F / A$ & داراى همسر & \\
\hline$M r$ & IOT/S & IfF/A & $A \cdot / A$ & $91 / 6$ & بى همسر در اثر فوت (ييوه) & \\
\hline m & $17 \cdot 18$ & IMT/F & $g x / 9$ & str & بى همسر در ائر طلاق & وضعيت تأهل \\
\hline$\pi$ & $11 \%$ & INA & $1 . r / 1$ & $1.9 / 8$ & هرئز ازدواجنكرده & \\
\hline- & $\cdot 1 \cdot \Delta F^{*}$ & $.10 .8 * *$ & $.1+4 t$ &. $\mid+41$ & اتثاو بثا & \\
\hline 19V0 & $1.9 / 1$ & $111 / \Delta$ & $A V / r$ & VNA & بي سواد & \\
\hline qVA & ITNY & MEN & eq/ & $e r / r$ & ابتدايي & \\
\hline Mr & $1+4 / 1$ & $\mid+\% 1$ & $A r / q$ & $A \cdot R$ & راهنمايى & سطح تحصلات \\
\hline TMA & $111 / 4$ & $1.9 / 8$ & $\Delta F / \&$ & $A M / F$ & متوسطه يا دييلم & \\
\hline lar & $1+r / r$ & $1 \cdot r / r$ & $9+18$ & $9 \% / \%$ & دانشكاهى & \\
\hline- & $.1 .24=*$ & $.1+A r=0$ & .1 .9900 & $.1+11^{\prime \prime m e *}$ & اتاو بتا & \\
\hline ENA & $91 / 1$ & $9 \% / V$ & $81 / N$ & ef. & شاغل & \\
\hline $1 f \cdot f^{2}$ & $111 / 8$ & $119 / \Delta$ & $9 \% / A$ & $97 / 9$ & با درآمد بلون كار & \\
\hline IITr & 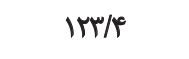 & $M \cdot / r$ & WA & AQ/8 & خائهدار & وضعيت فعاليت \\
\hline rqه & IMV/A & $110 / 9$ & Q⿻ & $90 / 1$ & ديكر & \\
\hline- & $. / N+\cdots$ & $+11 \cdot 1^{* * * *}$ & 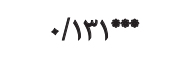 &.$/ 11 V^{m * *}$ & اثتا و بثا & \\
\hline ner & $I I r / \Delta$ & $\| 1 \% / \Delta$ & $9+/ \pi$ & $28 / 0$ & بايين & \\
\hline (זוTז & $110 /$ & $11 \% / 8$ & $\lambda F / 8$ & $A M / F$ & متوسط & \\
\hline pes & $118 / V$ & $\| 110 / \pi$ & Ma & AF/ & بالا & \\
\hline- & .1 .18 & $+\%+r$ & $.1 \cdot r g$ & $.1+\Delta \sigma^{* * *}$ & اتا و بتا & \\
\hline
\end{tabular}




\begin{tabular}{|c|c|c|c|c|c|c|}
\hline \multirow{2}{*}{ تعداد نمونه } & \multicolumn{2}{|c|}{ فعاليتهاي مذهبي } & \multicolumn{2}{|c|}{ مشاركت اجتماعي و فعاليتهائي } & & \\
\hline & $\begin{array}{l}\text { Adjusted for } \\
\text { Factors }\end{array}$ & Unadjusted & $\begin{array}{l}\text { Adjusted for } \\
\text { Factors }\end{array}$ & Unadjusted & & \\
\hline pre & $I T F / N$ & $1 r E / 0$ & $1 \cdot 1 / \Delta$ & $1 \cdot T / r$ & تنها & \\
\hline$r+p$. & $111 \% /$ & $11 \pi / 1$ & $A \varphi \%$ & $1 r / q$ & غيرتنها & وضعيث تنهايي \\
\hline- & $+1+\mu T^{*}$ & $+\mid+\Delta)^{-*}$ & $1.940=$ & $1+89=$ & اثأو بتا & \\
\hline MIO & $91 / 1$ & $q e / r$ & $e r / q$ & $n \neq / 1$ & بلى، دارد & \\
\hline$m+1$ & $118 / r$ & $110 / 1$ & AV/A & AV/T & خير، ندارد & نياز به مراقبت بهدليل \\
\hline- & .1 .98000 & $+(+\Delta)^{* *}$ & .1 .9 .0$. &.$/ \cdot M$ & اتا و بتا & \\
\hline \multirow{2}{*}{ TAIE } & \multicolumn{2}{|c|}{$\Delta / \cdot q^{* \cdots *}$} & \multicolumn{2}{|c|}{$\Delta M^{* \cdots *}$} & \multicolumn{2}{|c|}{ F Model } \\
\hline & \multicolumn{2}{|c|}{.$/ 1 A r(\Psi / Y \%)$} & \multicolumn{2}{|c|}{$\cdot / 19 \cdot(1 / 8 \%)$} & \multicolumn{2}{|c|}{$R\left(R^{2}\right)$} \\
\hline
\end{tabular}

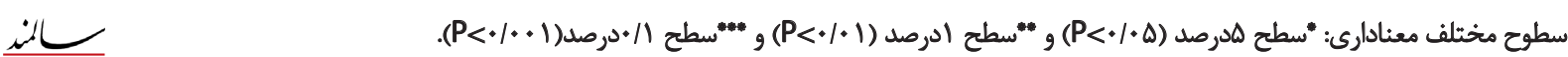

زمان اختصاصيافته سالمندان به كارهاى خانكى بدوندستمزد بود.

تحليل جندمتغيرى عوامل مؤثر بر زمان اختصاصيافته به

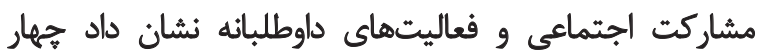

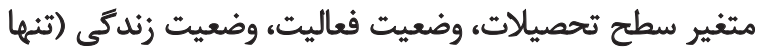

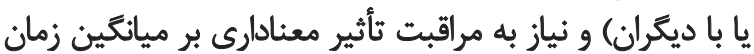

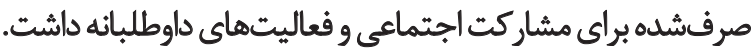

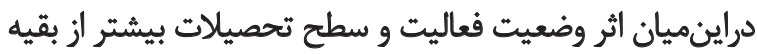

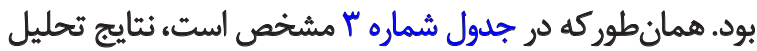

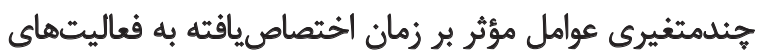

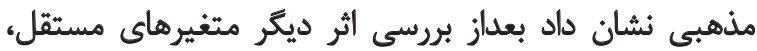

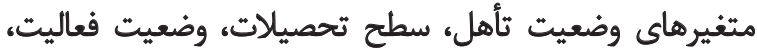

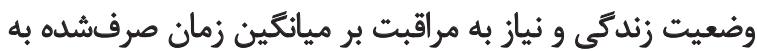

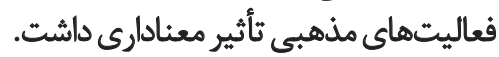

جدول شماره ץ نتايج آزمون MCA را درزمينه عوامل مؤثر

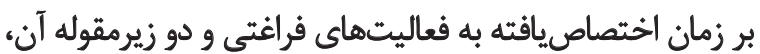

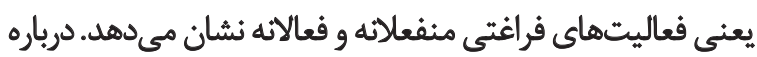

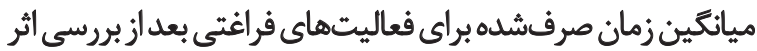

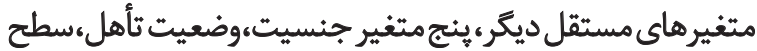

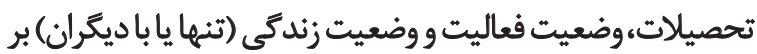

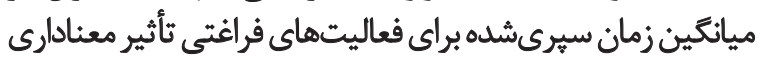

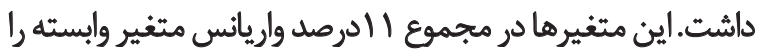

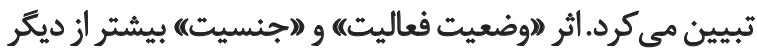

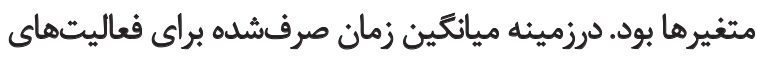

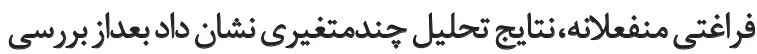

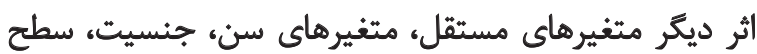

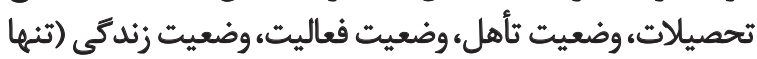

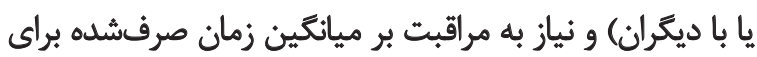

بعد از بررسى اثر ديكر متغيرهاي مستقل، جنسيت، وضعيت تأهل

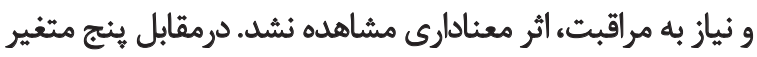

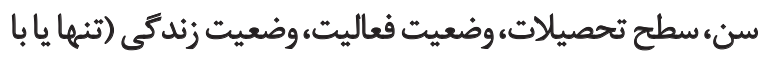

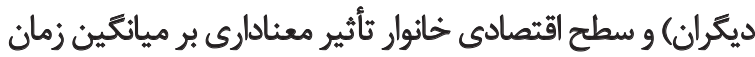

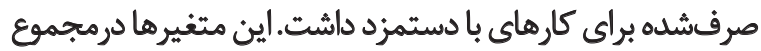

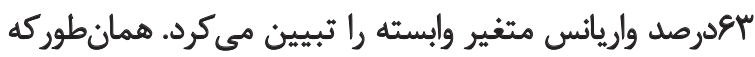

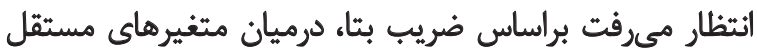

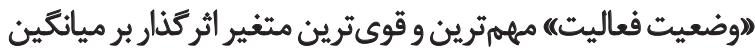

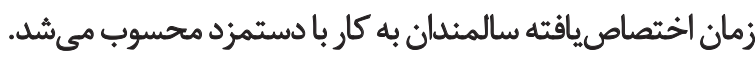
نتايج تحليل جندمتغيرى نشان داد متغيرهاى تحصيلات،

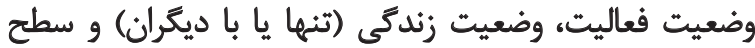

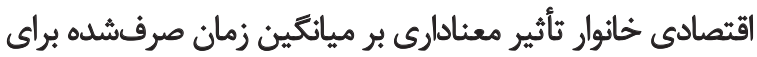

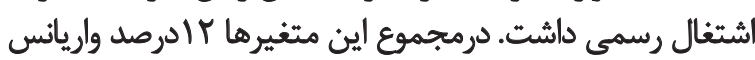

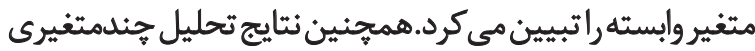

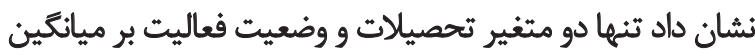

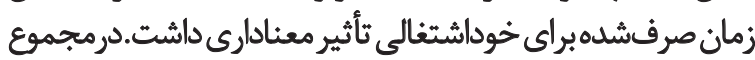

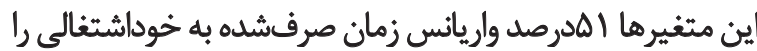

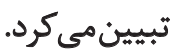

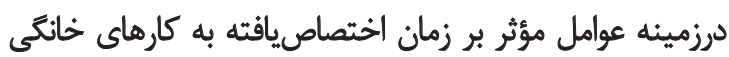

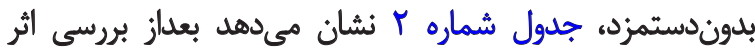

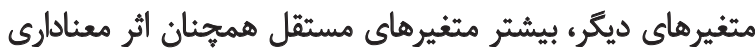

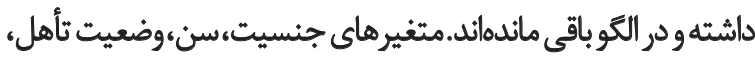

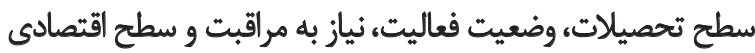

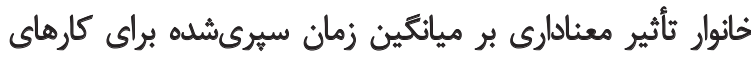

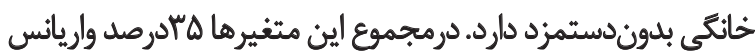

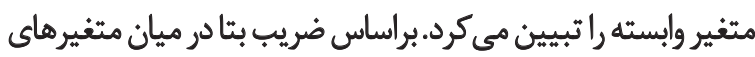
مستقل، "جنسيت) مهمثرين و قوى ترين متغير اثركذار بر ميانكين 
جدول F. همربستهاي اقتصادياجتماعى زمان اختصاصيافته به فعاليتهاي فراغتى (نتايج آزمون MCA).

\begin{tabular}{|c|c|c|c|c|c|c|c|c|}
\hline \multirow[b]{2}{*}{ تمونه } & \multicolumn{2}{|c|}{ فعاليتهاي فراغتى فعالائه } & \multicolumn{2}{|c|}{ فعاليتهاى فراغتى منفعلاثه } & \multicolumn{2}{|c|}{ فعاليتهاي مرتبط با اوقات } & & \\
\hline & 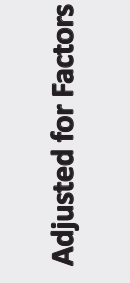 & 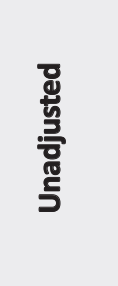 & 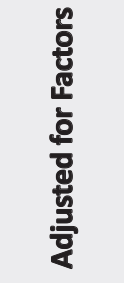 & 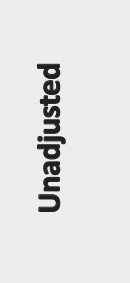 & 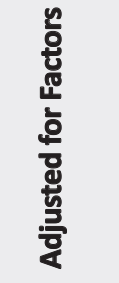 & 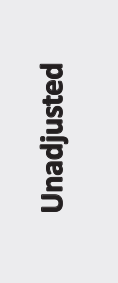 & & \\
\hline$|N|$ & $P \Psi \%$ & $\mu r / r$ & $r+\& / r$ & $r+1 / r$ & $r p q / r$ & $m p t / 4$ & 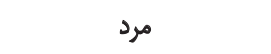 & \\
\hline $19 \Delta \Delta$ & $10 / \Lambda$ & $10 / 8$ & $|A| / 9$ & $\mathrm{AV} / \mathrm{P}$ & $19 \vee / V$ & $r \cdot r \%$ & نj & جنسيت \\
\hline- & orivere & $=/ T H+\cdots$ & $1 / \Lambda^{2=*}$ & $+1+4 \lambda^{* *}$ & ./Neran & 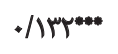 & اثتا و بثا & \\
\hline $111 \mathrm{r}$ & $r q / r$ & TNT & $19 r / 4$ & $\mid N \Delta / T^{m}$ & TrT/S & rir/s & 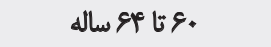 & \\
\hline VAr & $r+/ \Lambda$ & $r+/ r$ & WE/A & $|A r /|$ & rIV/e & $r \mid r / r$ & هوتا 9ع ساله & \\
\hline ner & $M / \Delta$ & $M / V$ & $191 / 9$ & $19 r / 9$ & TrQ/A & TrVIS & Vi & 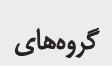 \\
\hline$p q \Delta$ & $r q / r$ & $r \cdot / r$ & $19 v / 4$ & r.r/g & TrEls & $m \pi / 9$ & ساله VI Vi va & سنى \\
\hline rqa & $r e / r$ & TEN & riv/e & TrNT & mer/e & TFQ/. & مي ساله و بيشتر & \\
\hline- & $.1 . \pi$ &.$\% F r$ & $1+\Delta 9^{*}$ &.$/ 110^{* * * *}$ & $.1+48$ & $\cdot 11 \cdot r^{+* * *}$ & اتثا وبثا & \\
\hline$T \Delta M$ & $r \cdot 10$ & $m / \Delta$ & $19 \cdot 11$ & $191 \%$ & $M M / r$ & $m$ mis & داراى همسر & \\
\hline Mr & $r q / p$ & $r \cdot / r$ & $r \cdot P / V$ & $r \cdot \varphi / m$ & $m \in /$ & MTEN & بي همسر در اثر فوت (ييوه) & \\
\hline$\pi$ & $1 \mathrm{ir/s}$ & $19 / 9$ & 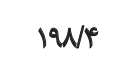 & $r+. / T$ & rIT/. & rIVI. & بى همسر در اثر طلاق & وضأهل \\
\hline ir & $n / s$ & $W / N$ & $m / 9$ & roq/ & $\Gamma \Delta / \Delta$ & me/q & هركز ازدواجنكرده & \\
\hline - & $+1 \cdot \Delta^{*}$ & $\cdot N \cdot$ &.$/ \Delta \Delta \Delta^{*}$ & .1 .49$. & $.1 .81^{+\infty}$ &.$/ . \mu f$ & اثتا و بتا & \\
\hline 19vo & $r \Delta / f^{f}$ & $\mathrm{rl} / \mathrm{H}$ & $M$. & $191 / N$ & rIr/s & TIT/S & بىسواد & \\
\hline q४^ & $r$ & $M / T$ & $r \cdot+/ r$ & $19 \Delta /$. & MTIS & rrq/1 & ايثدائى & \\
\hline Mr & rNq & $\mathrm{PV} / \mathrm{.}$ & TIV/T & TIR/T & $T A \& / T$ & $T E \cdot T$ & راهنمايى & سطع \\
\hline MrA & $P V / q$ & QNA & IWT & $\mathrm{IA} / \mathrm{A}$ & rWE & 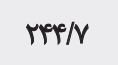 & متوسطه يا دييلم & تحصيلات \\
\hline IAT & PV/G & $81 / 7$ & TYNA & $m M / q$ & TVE/A & TAD/S & دانشكاهي & \\
\hline- & $. / 11)^{n+m}$ & $\cdot r+r=$ & .1 .10 & $.1 \cdot \Delta$ & $\cdot \Lambda \cdot r^{-\infty}$ &.$/ 11 \mathrm{~A}^{* * *}$ & اتثا و بثا & \\
\hline ENA & epr & $1 \% / 1$ & IFNA & $10 \mathrm{H} / \Delta$ & $1 \Delta r / \pi$ & $I V \cdot / r$ & 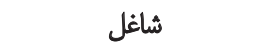 & \\
\hline $1 f+4$ & $+M$ & FNT & $r \cdot N A$ & $M / D / 0$ & $M F q / q$ & ret/A & داراى درآمد بلون كار & \\
\hline IITr & $r q / 8$ & $\mid f / *$ & $19 \& / V$ & $|A+1|$ & TrE/r & $194 / 1$ & خانهدار & فوضعيت \\
\hline rqם & $r+19$ & $r v / r$ & Trg/D & MFV/O & rEV/F & TAF/ & ساير & \\
\hline - & 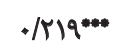 & •/TOA &.$/ 198000$ &.$/ 19 \gamma^{* 0 *}$ & . & $+/ \pi r^{* \cdots *}$ & اتأ و بتا & \\
\hline
\end{tabular}




\begin{tabular}{|c|c|c|c|c|c|c|c|c|}
\hline \multirow[b]{2}{*}{ تمونه } & \multicolumn{2}{|c|}{ فعاليتهاى فراغتى فعالاثه } & \multicolumn{2}{|c|}{ فعاليتهاى فراغتى منفعلاته } & \multicolumn{2}{|c|}{ فعاليتهاى مرتبط با اوقات } & & \\
\hline & 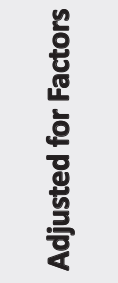 & 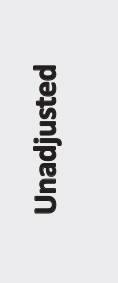 & 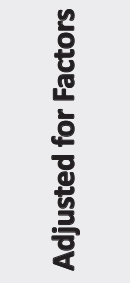 & 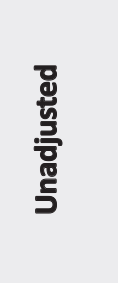 & 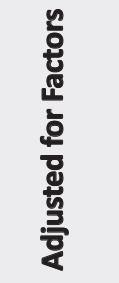 & 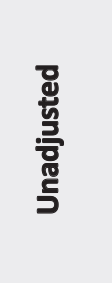 & & \\
\hline ner & $r \Delta / q$ & $M E / F$ & $r+V / A$ & $199 / \pi$ & $m \cdot N$ & mrres & بايين & \\
\hline חיוT & $r q / 9$ & $r \cdot / A$ & $19 \% / \pi$ & $194 / 8$ & 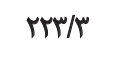 & $M T / \mu$ & متوسط & |قتصاديح \\
\hline Pfe & r\$/q & rg/q & $1199 / 2$ & $194 / K$ & MTH/ & $m+/$ & بالا & خانوار \\
\hline- & $.1 .4 q^{\circ}$ & $.1 \cdot \Delta \mathrm{A}^{* *}$ & $.1 . r V$ & .1018 & $.1 \cdot 19$ &.$/ \cdot 14$ & أتاو بِّا & \\
\hline pre & rNR & $M \in / q$ & $I V \cdot / A$ & $\mathrm{Ar} / \mathrm{A}$ & 19ข/1 & $r \cdot N A$ & تنهيا & \\
\hline$r \cdot f \cdot$ & $r+10$ & ry. & IWD & $198 / 5$ & Trq. & TrV/A & غيرتنها & تنهايي \\
\hline- &.$/ .14$ & $.1 \cdot m$ & $.1 .8 \Delta^{* * *}$ &.$/ \%$ & $.1 .80^{\circ * *}$ & $\cdot(\cdot p)^{*}$ & اتثا وبثا & \\
\hline mo & $19 / 1$ & $r \cdot 18$ & rarn & $\mathrm{m} \nabla / 9$ & $M / N$ & rq/Q & بلى، دارد & نياز به \\
\hline$M+1$ & $r \cdot / q$ & $r+/ 1$ & $19 \cdot 19$ & $119 / 4$ & $M M / A$ & $M+r$ & خير، ندارد & بهرلبي \\
\hline- & $1 .+5 a^{\circ *}$ & $.1 .49^{*}$ & $-N \cdot+\infty=0$ & - /1Fenew & $\cdot 1 \cdot \mathrm{VA}^{\infty * *}$ &.$/ 11 r^{m e n}$ & اثنا و بتا & ناتوائي \\
\hline \multirow{2}{*}{ rale } & \multicolumn{2}{|c|}{ ru/reane. } & \multicolumn{2}{|c|}{$1 . / 19+* *$} & \multicolumn{2}{|c|}{$W^{W}+\cdots$} & \multicolumn{2}{|c|}{ F Model } \\
\hline & \multicolumn{2}{|c|}{$\cdot / r \Delta \cdot(1 r / r \%)$} & \multicolumn{2}{|c|}{.$/ T \Delta A(\& / 8 \%)$} & \multicolumn{2}{|c|}{-/MTA(11) } & \multicolumn{2}{|c|}{$R\left(R^{2}\right)$} \\
\hline
\end{tabular}

L

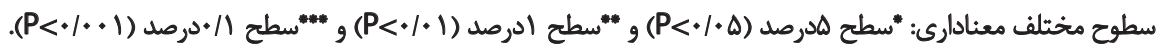

درمجموع • ادرصد واريانس متغير وابسته را تبيين مىكرد. براساس ضريب بتا در ميان متغيرهاى مستقل، (اسن و وضعيت

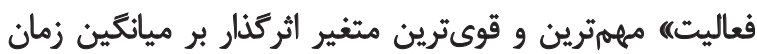

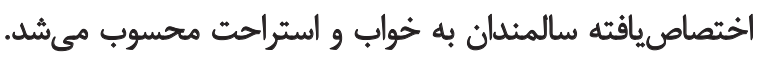

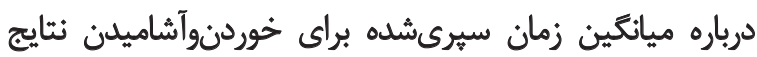

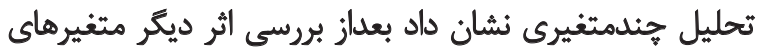

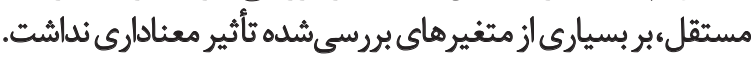

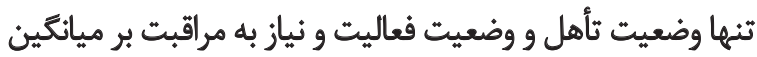

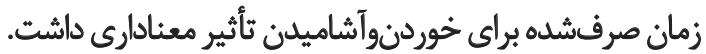

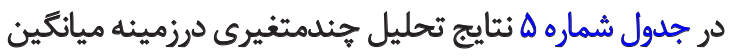

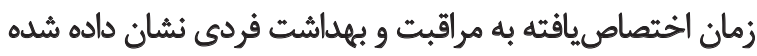

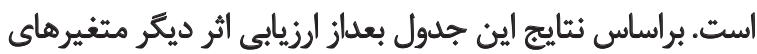

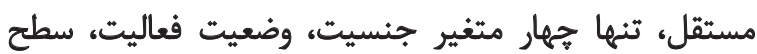

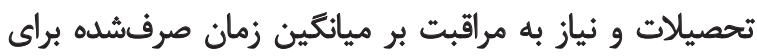

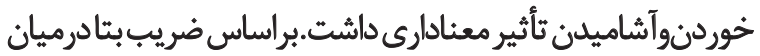

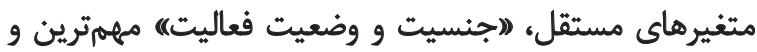

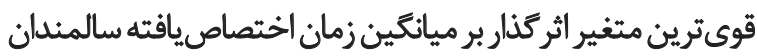

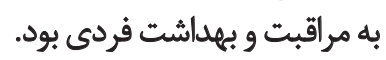

فعاليتهاى فراغتى منفعلانه تأثير معنادارى داشت. درباره ميانكين زمان اختصاصيافته به فعاليتهاي فراغتى

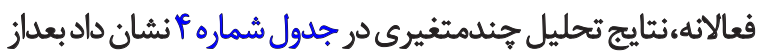

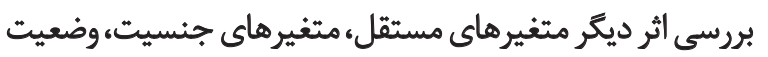

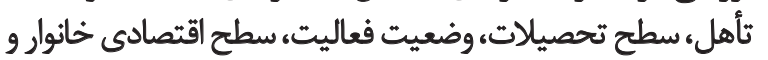

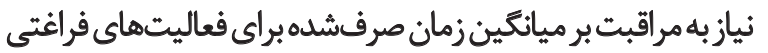

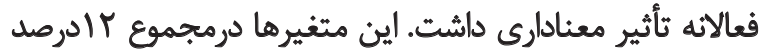

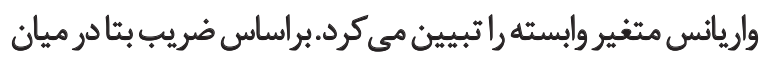

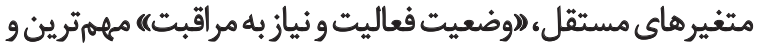

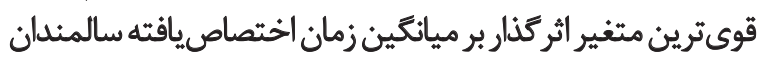
به فعاليتهاى فراغتى فعالانه بودي.

در جدول شماره ه نتايج آزمون MCA درزمينه عوامل مؤثر بر زمان

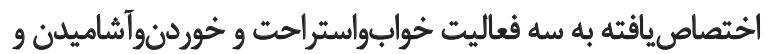

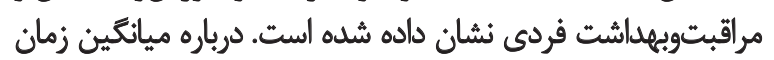

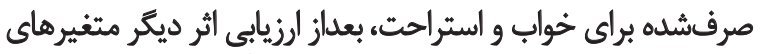

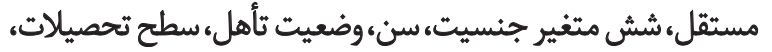

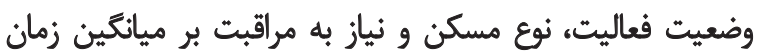

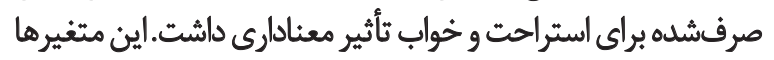


جدول ه. همبستههاى اقتصادىاجتماعى زمان اختصاصيافته به خوابواستراحت و خوردنوآشاميدن و مراقبتوبهداشت فردى (نتايج آزمون MCA).

\begin{tabular}{|c|c|c|c|c|c|c|c|c|}
\hline \multirow[b]{2}{*}{ تعد|اد نمونه } & \multicolumn{2}{|c|}{ مراقبتوبهداثشت فردى } & \multicolumn{2}{|c|}{ خوردنوأشاميدن } & \multicolumn{2}{|c|}{ خوابسواسترامث } & & \\
\hline & 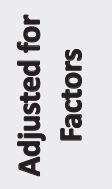 & 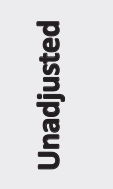 & 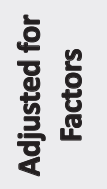 & 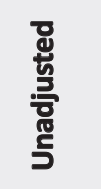 & 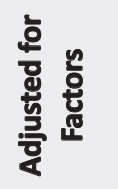 & 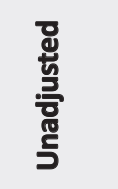 & & \\
\hline$|A|$ & $e V / F$ & EVIV & $118 / 9$ & $11 \mathrm{~V} /$ & $\Delta M r / r$ & $\Delta r \cdot / r$ & مرد & \\
\hline $1 \varepsilon \Delta \Delta$ & $\Delta \& /$ & QH/V & $\| F / A$ & $\| F / N$ & $\Delta I V / \varphi$ & $\Delta M T / Y$ & ij & جنسيث \\
\hline- & •ハ. & $\cdot / 1+\Lambda^{* * *}$ & $*+r$ &.$/+M$ & $.1 \circ \Delta \Delta^{*}$ & $*+H^{*}$ & ائا و بتا & \\
\hline$M \pi$ & $8 \cdot 1 /$ & $\Delta V / T$ & $11 \Delta / \mu^{\mu}$ & $110 / 1$ & $\Delta \cdot \Delta / N$ & $r q \Delta / F$ & . عأ بع ساله & \\
\hline VAr & $\Delta q / 9$ & ANE & $11 \%$ & $11 \Delta / A$ & ATI/S & $\Delta I T / N$ & هو تا 99 ساله & \\
\hline ner & $\Delta Q /$. & $8+18$ & $11 \Delta / 4$ & $11 \Delta / \mathrm{V}$ & DTA/S & $\Delta \omega+/$ & Vtr. & كروه هاى \\
\hline 190 & $9 \mathrm{Q} / 1$ & EN. & $11 W F$ & $|W|$ & DFV/D & $\Delta \Delta \& / 9$ & Vا Vا VA VA & سنى \\
\hline mq & $8 r / 9$ & $89 / 9$ & $110 /$. & $\| \Delta / A$ & $\Delta \& \Delta / 9$ & Q91/1 & مي ماله و بيشتر & \\
\hline- &.$\% \pi$ & $+/ * n)^{* *}$ & $+H$ & $.1 \cdot 11$ & . & $. / r+q \cdots * *$ & اثتا و بتا & \\
\hline rAM & $q \cdot N$ & $81 /$. & $118 / 9$ & IIV/8 & $\Delta r+/ r$ & QIF $/$ & داراى همسر & \\
\hline Mr & $g r / r$ & $81 / 0$ & $11 \cdot 10$ & $111 / \pi$ & $\Delta F I / 9$ & $\Delta \& 1 / \Delta$ & بى همسر در اثر فوت (ييوه) & \\
\hline$\pi$ & $\varepsilon \cdot \pi$ & $\Delta V / V$ & $111 / r$ & $11 \%$ & $19 q 1 / 1$ & $e q r / \Delta$ & بى همسر در اثر طالاق & وضعيت تأهل \\
\hline m & $V \Delta /{ }^{\circ}$ & $v \cdot / f$ & $1.1 / 1$ & $1 .+18$ & $\Delta f^{e} / / 9$ & $\Delta r V N$ & هركز ازدواجنكرده & \\
\hline- & $.1 .1 \mathrm{~V}$ & .1 .11 & $.1 .9 T^{\prime \prime}$ &.$(\cdot \Delta)^{e *}$ & $.1 .8 \mathrm{~V}^{* *}$ &.$/$ ferman & اتئا و بتا & \\
\hline 19va & RT/A & st/ & $118 / 9$ & $118 / \pi$ & $\Delta M r / \Delta$ & are/A & بي سواد & \\
\hline จ४А & $\Delta q / V$ & $8 \cdot 11$ & $11 r / 9$ & $11 F / \pi$ & $019 / 1$ & $\Delta 1 r / r$ & ابتداييى & \\
\hline Mr & $9 \cdot 1 /$ & $g \pi / \varphi$ & $M \pi / r$ & $11 \% / 9$ & $\Delta \cdot V / V$ & $\Delta \cdot V /$. & راهنمايي & حaw \\
\hline Tra & $\Delta 1 / r$ & $\Delta \Delta / \Delta$ & llels & $119 / 1$ & $\Delta \mid V /$. & $\Delta r r / q$ & متوسطه يا دييلهم. & تحصيلات \\
\hline lar & $\Delta V / 8$ & $\Delta V / T$ & IWS & $119 / \pi$ & $p q \cdot / q$ & paq. & دانشكاهي & \\
\hline- & $.1 .09^{\circ}$ & .1 .49 & 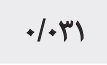 &.$\%$ rA & $+1+V \mathrm{~A}^{* 0 * *}$ & $.1 \cdot 90^{=0 *}$ & اتا و بتا & \\
\hline SNA & $\Delta Y N$ & $\Delta \& / A$ & $1.9 / \pi$ & $11 \cdot 14$ & $\mathrm{PV} / \mathrm{A}$ & $P e r / r$ & 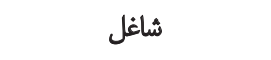 & \\
\hline $1 f \cdot f$ & SNT & $e q / 9$ & IIVN & $I I V / N$ & $\Delta+T / q$ & $\Delta F q / r$ & با درآمد بلون كار & \\
\hline IITr & $\Delta \otimes / V$ & $r q / Y$ & IIV/r & $11 \Delta / A$ & $\Delta \Psi / q$ & $\Delta R r / q$ & خانهدار & وضعيت \\
\hline rqd & sV/a & 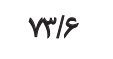 & $118 / 9$ & $119 / 1$ & $\Delta F I N$ & $\Delta V+/ \lambda$ & ساير & \\
\hline- & $+/ 1+\gamma^{+\infty+*}$ & +/Nequ* & $.1 .81^{\circ}$ & $+1+\Delta+\infty$ & +MAcen & D/Mmesen & اتاو بتا & \\
\hline nev & $8 \cdot / r$ & ST/F & $119 / \Delta$ & $\mid I V / 8$ & $019 / 8$ & arge & بايين & \\
\hline rזr & $81 / r$ & $81 / 1$ & $\| \Delta / f$ & $\| \Delta / V$ & $\Delta r V / a$ & $\Delta r \Delta / 9$ & متونسط & اقتصادي \\
\hline peg & $g r / r$ & $\Delta \mathcal{N Q}$ & $11 \mathrm{r} / \mathrm{g}$ & $11 r / q$ & $\Delta r \Delta / r$ & $\Delta W Q$ & بالا & خائوار \\
\hline- & $.1 . .9$ & .1 .18 & $.1 .4 q$ &.$/ . r$ & \% & 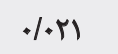 & اثتا و بتا & \\
\hline
\end{tabular}




\begin{tabular}{|c|c|c|c|c|c|c|c|c|}
\hline \multirow[b]{2}{*}{ ت تعداد نمونه } & \multicolumn{2}{|c|}{ مراقبتوبيهداشت فردى } & \multicolumn{2}{|c|}{ خوردنوآشاميدن } & \multicolumn{2}{|c|}{ خوابواستراحت } & & \\
\hline & 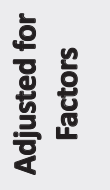 & 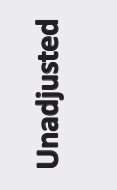 & 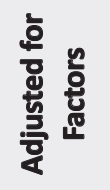 & 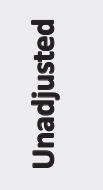 & 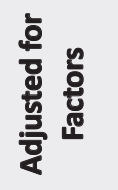 & 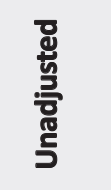 & & \\
\hline pve & 8.11 & $8 / 8$ & $11 \% /$ & $11 . / 4$ & are// & $\Delta f \mid / T^{\circ}$ & تنها & \\
\hline$p+\varphi$ & $81 / \pi$ & $91 / 1$ & $111 / \Delta$ & $119 N$ & ATE/ & $\Delta r r / \varphi$ & غيرتنها & تنهيايع \\
\hline- & $+1 \cdot \cdot v$ & /..r & . $1 . \% 9$ & $+1+\varphi \cdot$ & 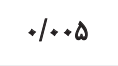 & 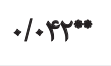 & اتاو بتا & \\
\hline mo & $e q / r$ & $v \Delta / *$ & $1 \pi r / r$ & $\mid r r / q$ & DSNY & $9 . r / 1$ & بلى، دارد & \\
\hline$\pi+1$ & 8.18 & $9 . / \pi$ & $11 \Delta /{ }^{4}$ & $11 \Phi / 4$ & QHTH & $\Delta r \cdot N$ & خير، ندارد & بياز به مراقبت \\
\hline- &.$\% \mu^{*}$ & $.1+\Delta \Delta^{\cdots \cdots}$ & $1 .+\Delta^{*}$ & $+\%$ & $+1+V Q^{\circ+\infty}$ & $. / 1 r r+e$ & اتاو بتا ل & \\
\hline rale & $\begin{array}{r}r \\
. / 1 V\end{array}$ & & & & in & & & \\
\hline
\end{tabular}

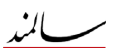

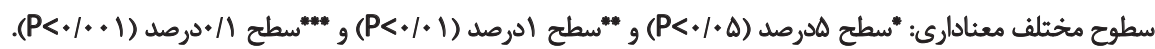

كانادايى زمان بيشترى را براى فعاليتهاى فراغتى و توليدى و

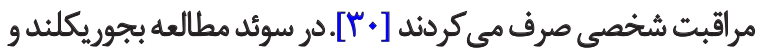

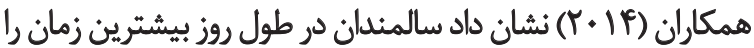

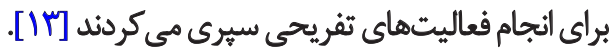
علاومبر متوسط زمانهاى اختصاصدادهشده براى فعاليتهاى

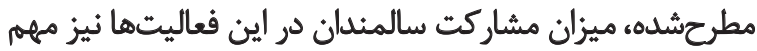

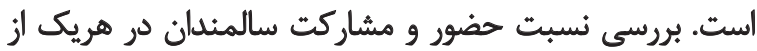

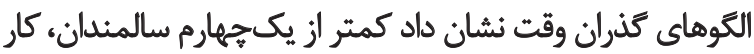

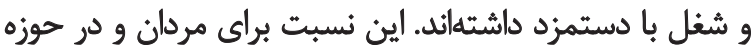

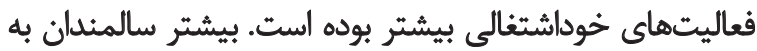

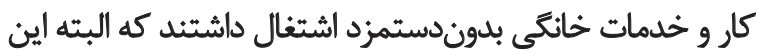

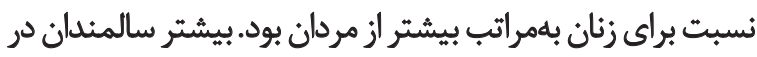

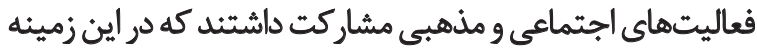

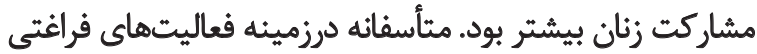

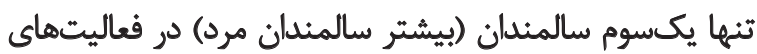

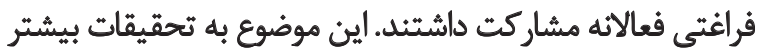

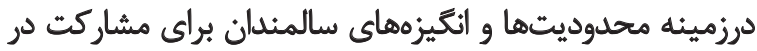
الكوهاى كذران وقت وفعاليتهاى فراغتى فعالانه نياز دارد.

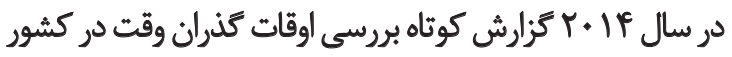

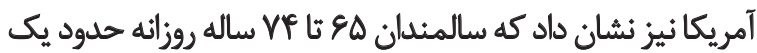

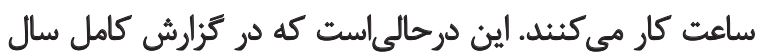

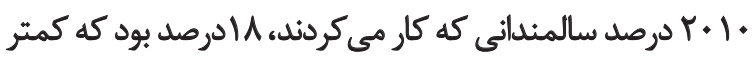

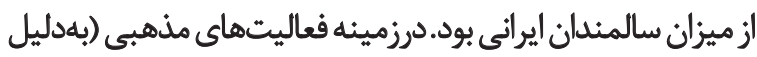

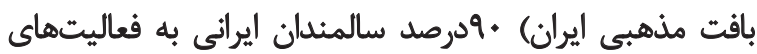

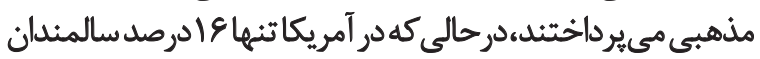

الكوهاى كذران وقت براساس جرخه زندگى تغيير مى كثند و

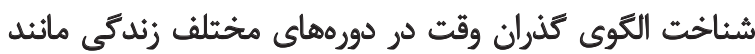

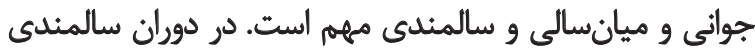

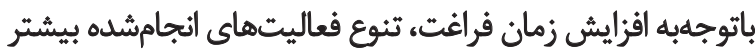

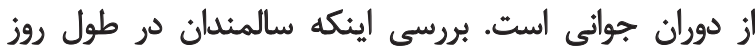

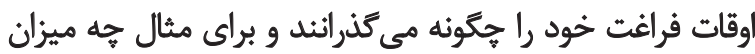

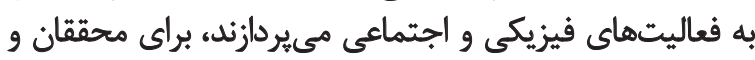

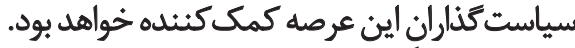

يافتهاي مطالعه نشان داد بيشترين ميانكين زمان

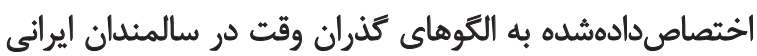

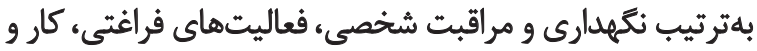

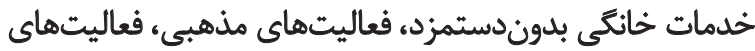

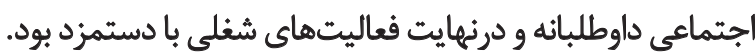

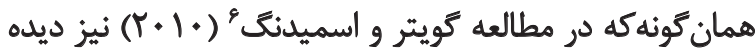

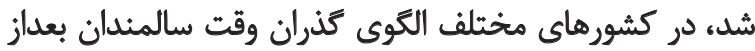

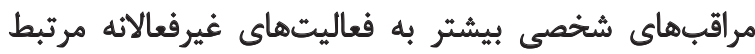

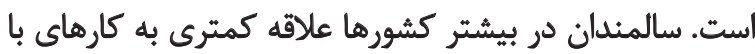

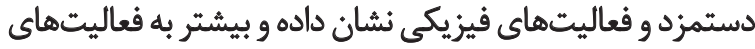

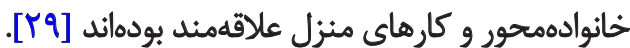
در كانادا مككنون (1991) مطالعهاى انجام داد كه متوسط زمان

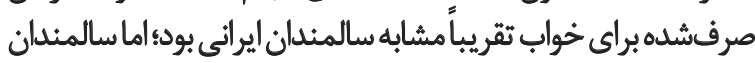

6. Gauthier and Smeeding 
داوطلبانه نيز بهطورمعنادارى افزايش يافت. مطالعات ديكر نيز

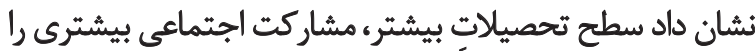

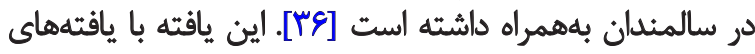
مطالعات ديكر همراستا بود. كَفتنى است سطح تحصيلات كمتر،

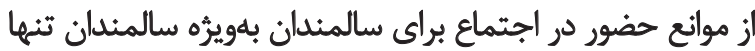

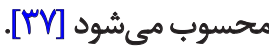

وضعيت فعاليت يكى از مهمثرين متغيرهاي اثركذار بر الكوهاى

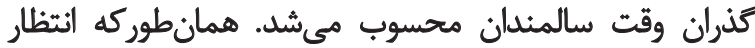

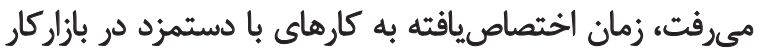

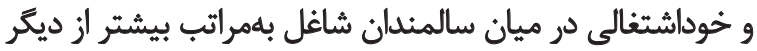

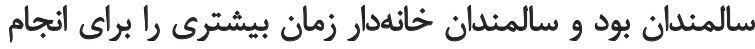

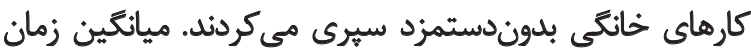
صرفشده براى مشاركت اجتماعى و فعاليتهائ فراني فراغتى فئى فعالانه

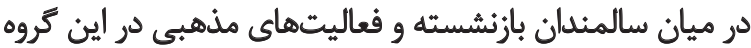

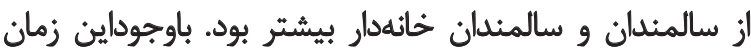

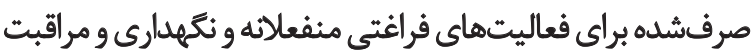

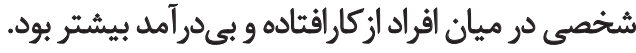
وضعيت اقتصادى خانوار يكى ديكًر از عواملى بود كه بهاطورمعناداد

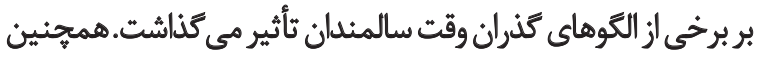

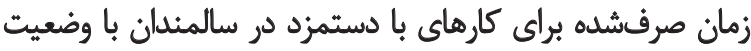

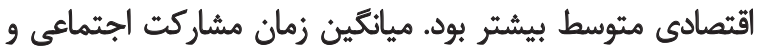

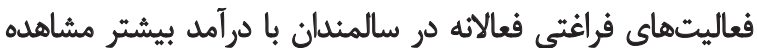

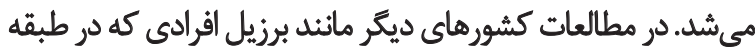

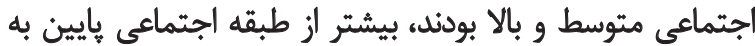

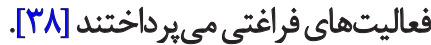

وضعيت زندكى سالمندان (تنها يا با ديكران)، موضوعى بود كه

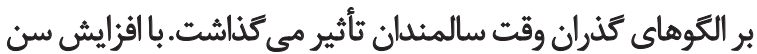

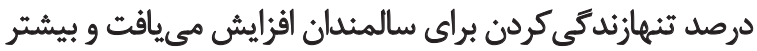

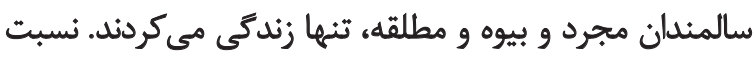

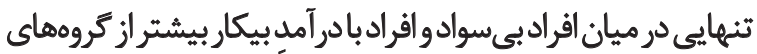

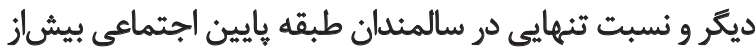

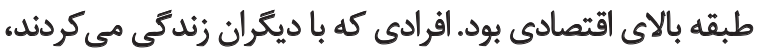

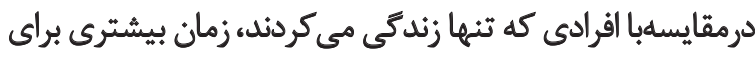

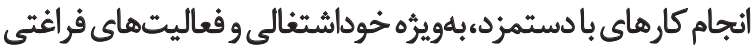

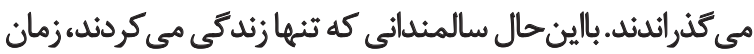

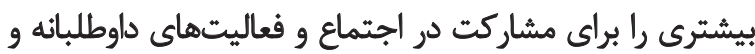
مذهبى و خوابيدن و استراحت صرف مي كردند.

براساس تحقيقات حضور سالمندان تنههادر اجتماع در فعاليتهاى

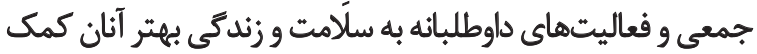

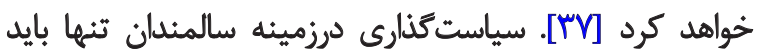

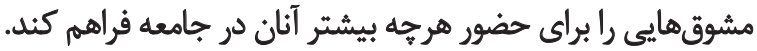

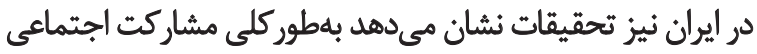

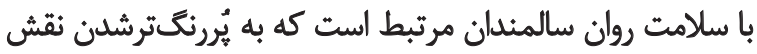

در فعاليتهاي مذهبى حضور داشتند كه بلهورت شركت در مراس

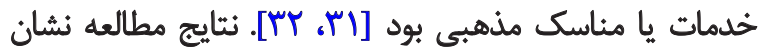

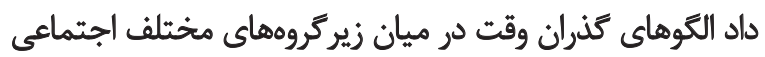

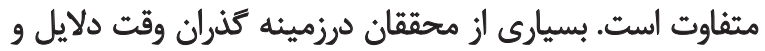

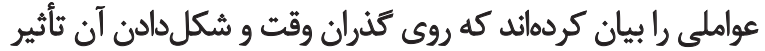

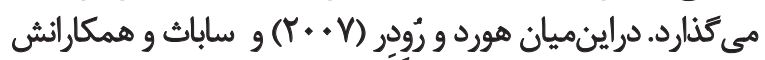

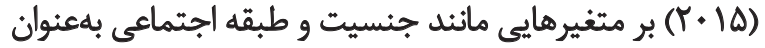

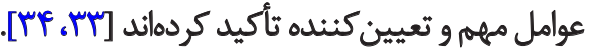

يافتهاي مطالعه حاضر نيز از تفاوتهاى جنسيتي محسوس در

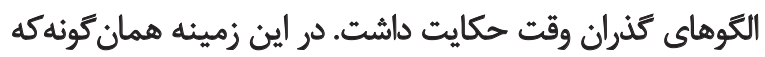

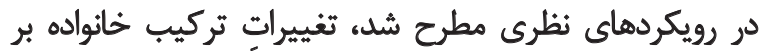

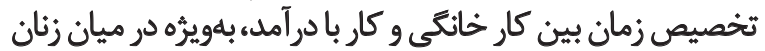

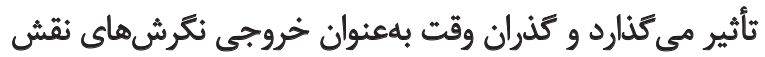

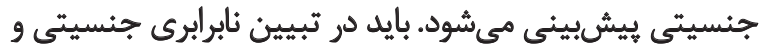

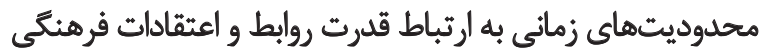

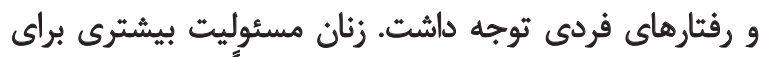

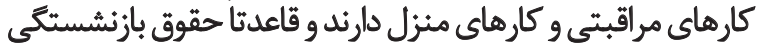

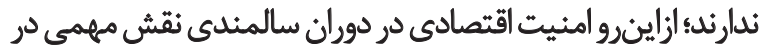

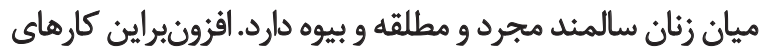

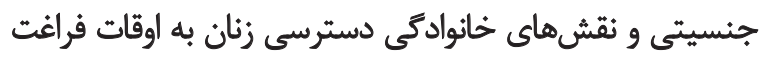

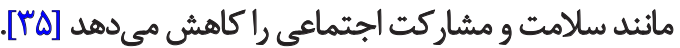
در بررسى رابطه ديكر متغيرهاى اجتماعى جمعيتشناختى با

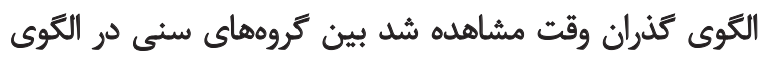

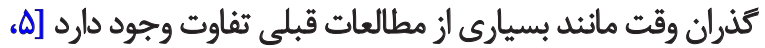

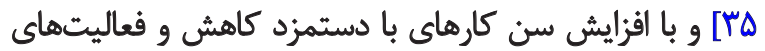

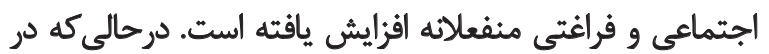

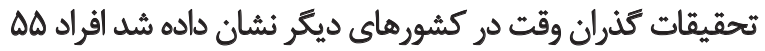

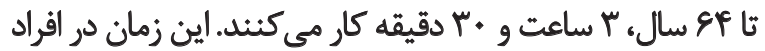

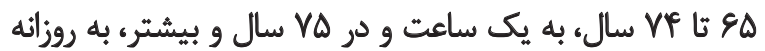

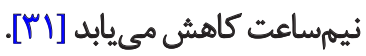

وضعيث الكوى كذران وقت در ميان سالمندان براساس "وضعيت

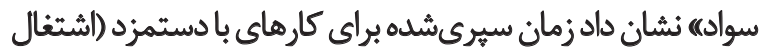

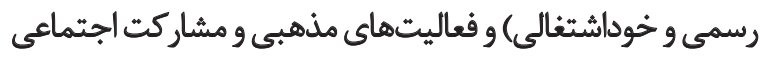

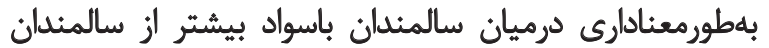

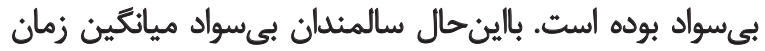

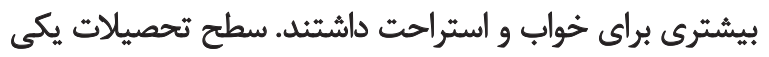

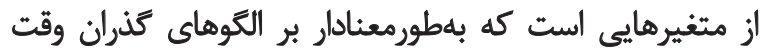

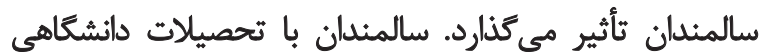

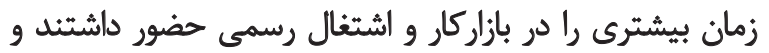

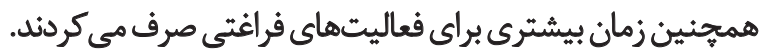

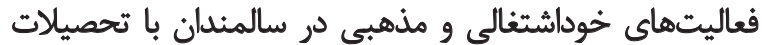

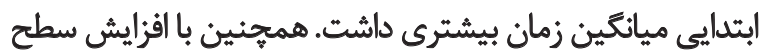

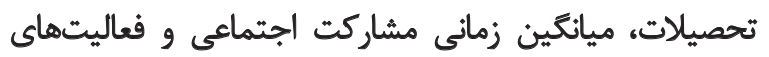




\section{References}

[1] Pruchno RA, Rose MS. Time use by frail older people in different care settings. Journal of Applied Gerontology. 2002; 21(1):5-23. doi: $10.1177 / 0733464802021001001$

[2] Galay K. Patterns of time use and happiness in Bhutan: is there a relationship between the two? Japan: Institute of Developing Economies Publication; 2007.

[3] Statistical Center of Iran. [Iranian's time use survey (Persian)]. Tehran: Statistical Center of Iran Publication; 2009.

[4] Fleming R, Spellerberg A, Zealand SN. Using time use data: a history of time use surveys and uses of time use data. Wellington: Statistics New Zealand; 1999.

[5] Gauthier AH, Smeeding TM. Time use at older ages crossnational differences. Research on Aging. 2003; 25(3):247-74. doi: $10.1177 / 0164027503025003003$

[6] Piekkola H. Demographic aspects of ageing and time use in a set of European countries. Finland: Research Institute of the Finnish Economy; 2004.

[7] Beblo M. Bargaining over time allocation: economic modeling and econometric investigation of time use within families. New York: Springer Science \& Business Media; 2001.

[8] Killingsworth MR. Labor supply. New York: Cambridge University Press; 1983

[9] Becker GS. A theory of the allocation of time. Economic Journal. 1965; 75(29):493-517. doi: 10.2307/2228949

[10] Sadeghi R, Balali E, Mohammadpur A. [Social inequality in leisure time (Persian)]. Social Welfare. 2011; 10(39):357-89.

[11] McKay J. Leisure and social inequality in Australia. Journal of Sociology. 1986; 22(3):343-67. doi: 10.1177/144078338602200301

[12] Binstock RH, George LK, Cutler SJ, Hendricks J, Schulz JH. Handbook of aging and the social sciences. New York: Academic Press; 2011.

[13] Björklund C, Gard G, Lilja M, Erlandsson LK. Temporal patterns of daily occupations among older adults in Northern Sweden. Journal of Occupational Science. 2013; 21(2):143-60. doi: $10.1080 / 14427591.2013 .790666$

[14] Cornwell B. Age trends in daily social contact patterns. Research on Aging. 2011; 33(5):598-631. doi: 10.1177/0164027511409442

[15] Espinel PT, Chau JY, van der Ploeg HP, Merom D. Older adults' time in sedentary, light and moderate intensity activities and correlates: application of Australian time use survey. Journal of Science \& Medicine in Sport. 2014; 18(2):161-66. doi: 10.1016/j. jsams.2014.02.012

[16] Gauthier AH, Furstenberg FF. The transition to adulthood: a time use perspective. Annals of the American Academy of Political \& Social Science. 2002; 580(1):153-71. doi: $10.1177 / 0002716202580001007$

[17] Gray A. The changing availability of grandparents as carers and its implications for childcare policy in the UK. Journal of Social Policy. 2005; 34(4):557-77. doi: 10.1017/s0047279405009153

[18] Jowsey T, Yen L, Mathews P. Time spent on health related activities associated with chronic illness: a scoping lit-
مشاركت اجتماعى در سياست كذارى ها نياز دارد [1؟].

$$
\text { تئيجدكيرى نهايى }
$$

باتوجهبه مطالب بيانشده مي توان نتيجه كرفت مجموعهاي

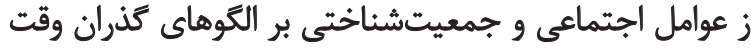

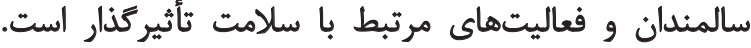

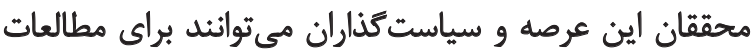

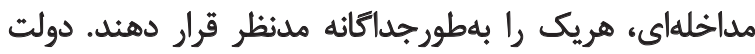

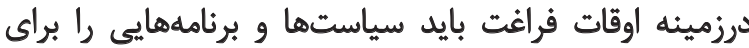

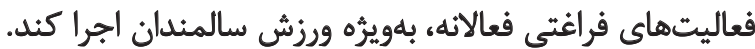

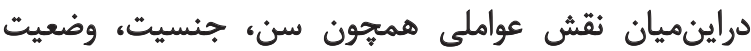

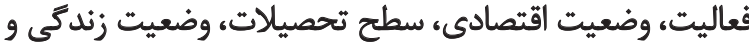

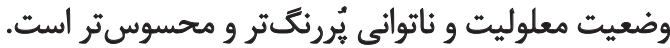

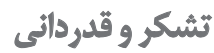

اين مطالعه بركرفته از طرح بروهشى با همين عنوان است كه

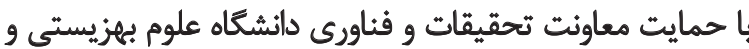

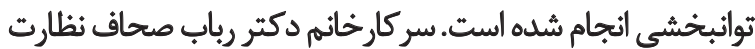

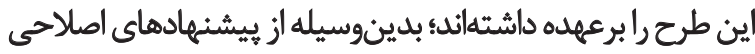
ايشان در حين انجام اين مطالعه تشكر و قدردانى مي ئشود. 
erature review. BMC Public Health. 2012; 12(1):1044-056. doi: 10.1186/1471-2458-12-1044

[19] Kellly J. Activity and ageing: challenge in retirement in work leisure and well being. London: Routledge Press; 1997.

[20] Larson R. Thirty years of research on the subjective well-being of older Americans. Journal of Gerontology. 1978; 33(1):109-25. doi: 10.1093/geronj/33.1.109

[21] Larson R, Zuzanek J, Mannell R. Being alone versus being with people: Disengagement in the daily experience of older adults. Journal of Gerontology. 1985; 40(3):375-81. doi: 10.1093/geronj/40.3.375

[22] Marcum CS. Age differences in daily social activities. Research on Aging. 2012; 35(5):612-40. doi: 10.1177/0164027512453468

[23] Velarde M, Herrmann R. How retirement changes consumption and household production of food: Lessons from German time-use data. Journal of the Economics of Ageing. 2014; 3:1-10. doi: 10.1016/j.jeoa.2013.12.003

[24] Sun J. Chinese older adults taking care of grandchildren: practices and policies for productive aging. Ageing International. 2013; 38(1):58-70. doi: 10.1007/s12126-012-9161-4

[25] Rahimi A, Anoosheh M, Ahmadi F, Foroughan M. [Tehranian elderly people's experiences about leisure time activities and recreations (Persian)]. Iranian Journal of Ageing. 2010; 5(1):61-77.

[26] Heshmati H, Asnashari R, Khajavi S, Charkazi A, Babak A, Hosseini SG, et al. [Life style of elderly in Kashmar, Iran 2012 (Persian)]. Journal of Research Development in Nursing \& Midwifery. 2014; 11(1):39-50.

[27] Goudarzi AM, Najimi A. [Healthy lifestyle of the elderly: a ccross-sectional study (Persian)]. Health System Research. 2013; 8(4):581-57.

[28] United Nations. Guide to producing statistics on time use: measuring paid and unpaid work. New York: United Nations Publication; 2005.

[29] Gauthier AH, Smeeding TM. Historical trends in the patterns of time use of older adults. In: Tuljapurkar B, Shripad A, Ogawa F, Naohiro H, Gauthier R, Anne H, editors. Ageing in Advanced Industrial States: New York: Springer; 2010, p. 289-310.

[30] McKinnon AL. Time use for self care, productivity, and leisure among elderly Canadians. Canadian Journal of Occupational Therapy. 1992; 59(2):102-10. doi: 10.1177/000841749205900206

[31] Bureau of Labor Statistics. American time use survey. New York: United States Department of Labor; 2010

[32] Bureau of Labor Statistics. American time use survey: 2014 results. New York: United States Department of Labor; 2015.

[33] Hurd MD, Rohwedder S. Time-use in the older population: variation by socio-economic status and health. In: Robert F, Belli F, Stafford P, Duane F, editors. Using Calendar and Diary Methodologies in Life Events. New York: RAND Labor and Population; 2007, p. 1-35.

[34] Sabbath EL, Matz-Costa C, Rowe JW, Leclerc A, Zins M, Goldberg $\mathrm{M}$, et al. Social predictors of active life engagement a timeuse study of young-old French adults. Research on Aging. 2015; 38(8):864-93. doi: 10.1177/0164027515609408
[35] Sayer L, Gornick J. Older Adults: International Differences in Housework and Leisure. Social Indicators Research. 2009; 93(1):215-18. doi 10.1007/s11205-008-9376-7

[36] Cachadinha C, Costa Branco De Oliveira Pedro JA, Carmo Fialho J. Social participation of community living older persons: Importance, determinants and opportunities. Paper presented at: The $6^{\text {th }}$ International Conference on Inclusive Design: The Role of Inclusive Design in Making Social Innovation Happen; 2011 Apr 18-20; London, UK.

[37] Goll JC, Charlesworth G, Scior K, Stott J. Barriers to social participation among lonely older adults: The influence of social fears and identity. PloS one. 2015; 10(2):e0116664. doi: 10.1371/journal. pone. 0116664

[38] Andrade AC, Peixoto SV, Friche AA, Goston JL, César CC, Xavier CC, et al. Social context of neighborhood and socioeconomic status on leisure-time physical activity in a Brazilian urban center: the BH Health Study. Cadernos de Saúde Pública. 2015; 31:136-47. doi: 10.1590/0102-311x00069514 